MODELING, IDENTIFICATION AND CONTROL, 2003, VOL. 24, NO. 3, 125-157

doi:10.4173/mic.2003.3.1

\title{
Subspace System Identification of the Kalman Filter*
}

\author{
DAVID DI RUSCIO $\dagger$ \\ Keywords: Identification methods, Subspace methods, Stochastic systems, \\ Sampled data systems, Linear systems.
}

\begin{abstract}
Some proofs concerning a subspace identification algorithm are presented. It is proved that the Kalman filter gain and the noise innovations process can be identified directly from known input and output data without explicitly solving the Riccati equation. Furthermore, it is in general and for colored inputs, proved that the subspace identification of the states only is possible if the deterministic part of the system is known or identified beforehand. However, if the inputs are white, then, it is proved that the states can be identified directly. Some alternative projection matrices which can be used to compute the extended observability matrix directly from the data are presented. Furthermore, an efficient method for computing the deterministic part of the system is presented. The closed loop subspace identification problem is also addressed and it is shown that this problem is solved and unbiased estimates are obtained by simply including a filter in the feedback. Furthermore, an algorithm for consistent closed loop subspace estimation is presented. This algorithm is using the controller parameters in order to overcome the bias problem.
\end{abstract}

\section{Introduction}

A complete subspace identification (SID) algorithm are discussed and derived in this paper. The derivation presented is different from the other published papers on subspace identification, Van Overschee and De Moor (19934), Larimore (1990), Viberg (1995) and Van Overschee (1995) and the references therein, because we are using general input and output matrix equations which describes the relationship between the past and the future input and output data matrices.

One of the contributions in this paper is that it is shown that the Kalman filter model matrices, including the Kalman gain and the noise innovations process, of a combined deterministic and stochastic system can be identified directly from certain projection matrices which are computed from the known input and output data, without solving any Riccati or Lyapunov matrix equations. This subspace method and results was presented without proof in Di Ruscio (1995) and Di Ruscio (1997). One contribution in this paper is a complete derivation with proof. A new method for computing the matrices in the deterministic part of the system is presented. This method has been used in the DSR Toolbox for Matlab, Di Ruscio (1996), but has not been published earlier.

Furthermore, it is pointed out that the states, in general (i.e. for colored input signals), only can be computed if the complete deterministic part of the model is known or identified first. This is probably the reason for which the state based subspace algorithms which are presented in the literature docs not work properly for

* This paper is an extended version of a paper with nearly the same title which was presented at The European Control Conference, ECC2001, Porto, Portugal 2001.

$\uparrow$ Telemark University College, Department of Process Automation, N-3914 Porsgrunn, Norway. Tel: +4735 5751 68, Fax: +4735 5752 50. E-mail: David.Di.Ruscio@hit.no 
colored input signals. The SID algorithm in Verhagen (1994) works for colored input signals. The stochastic part of the model is not computed by this algorithm. The N4SID algorithm in Van Overschee and De Moor (1994) works well and only for white input signals. The stochastic part of the model is computed by solving a Riccati equation. However, the robust modification in Van Overschee and De Moor (1995) works well also for colored input signals.

The rest of this paper is organized as follows. Some basic matrix definitions and notations are presented in Section 2. The problem of subspace identification of the states for both colored and white input signals is discussed in Section 3.1. The subspace identification of the extended observability matrix, which possibly is the most important step in any SID algorithm, are discussed in Section 3.2. It is proved that the kalman filter gain matrix and the noise innovations process can be identified directly from the data in Section 3.3. A least squares optimal method for computing the deterministic part of the combined deterministic and stochastic system is presented in Section 3.4. Some topics and remarks related to the algorithm are presented in Section 5. Numerical examples are provided in Section 6 in order to illustrate the behaviour of the algorithm both in open and closed loop. Some concluding remarks follows in Section 7.

\section{Notation and definitions}

\subsection{System and matrix definitions}

Consider the following state space model on innovations form

$$
\begin{aligned}
\bar{x}_{k+1} & =A \bar{x}_{k}+B u_{k}+C e_{k} \\
y_{k} & =D \bar{x}_{k}+E u_{k}+F e_{k}
\end{aligned}
$$

where $e_{k}$ is white noise with covariance matrix $E\left(e_{k} e_{k}^{T}\right)=I_{m}$. One of the problems addressed and discussed in this paper is to directly identify (subspace identification) the system order, $n$, the state vector $\bar{x}_{k} \in \mathbb{R}^{n}$, and the matrices $(A, B, C, D, E, F)$ from a sequence of known input and output data vectors, $u_{k}, \in \mathbb{R}^{r}$ and $y_{k}, \in \mathbb{R}^{m}$, respectively. A structure parameter, $g$, is introduced so that $g=1$ when $E$ is to be identified and $g=0$ when $E$ is a-priori known to be zero. This should be extended to a structure matrix $G$ with ones and zeroes, the ones pointing to the elements in $E$ which are to be estimated. This is not considered further here. Based on equations (1) and (2) we make the following definitions for further use:

\section{Definition 2.1. (Basic matrix definitions)}

The extended observability matrix, $O_{i}$, for the pair $(D, A)$ is defined as

$$
O_{i}^{\text {def }}=\left[\begin{array}{l}
D \\
D A \\
\vdots \\
D A^{i-1}
\end{array}\right] \in \mathbb{R}^{i m \times n}
$$

where the subscript $i$ denotes the number of block rows.

The reversed extended controllability matrix, $C_{i}^{d}$, for the pair $(A, B)$ is defined as

$$
C_{i}^{d} \stackrel{\text { def }}{=}\left[\begin{array}{llll}
A^{i-1} B & A^{i-2} B & \ldots & B
\end{array}\right] \in \mathbb{R}^{n \times i r}
$$


where the subscript $i$ denotes the number of block columns. A reversed extended controllability matrix, $C_{i}^{s}$, for the pair $(A, C)$ is defined similar to equation (4), i.e.,

$$
C_{i}^{s} \stackrel{\text { def }}{=}\left[\begin{array}{llll}
A^{i-1} C & A^{i-2} C & \ldots & C
\end{array}\right] \in \mathbb{R}^{n \times i m}
$$

i.e., with $B$ substituted with $C$ in equation (4). The lower block triangular Toeplitz matrix, $H_{i}^{d}$, for the quadruple matrices $(D, A, B, E)$

$$
H_{i}^{d} \stackrel{\text { def }}{=}\left[\begin{array}{lllll}
E & 0_{m \times r} & 0_{m \times r} & \ldots & 0_{m \times r} \\
D B & E & 0_{m \times r} & \ldots & 0_{m \times r} \\
D A B & D B & E & \ldots & 0_{m \times r} \\
\vdots & \vdots & \vdots & \ddots & \vdots \\
D A^{i-2} B & D A^{i-3} B & D A^{i-4} B & \ldots & E
\end{array}\right] \in \mathbb{R}^{i m \times(i+g-1) r}
$$

where the subscript $i$ denotes the number of block rows and $i+g-1$ is the number of block columns. Where $0_{m \times r}$ denotes the $m \times r$ matrix with zeroes. A lower block triangular Toeplitz matrix $H_{i}^{s}$ for the quadruple $(D, A, C, F)$ is defined as

$$
H_{i}^{d} \stackrel{\text { def }}{=}\left[\begin{array}{lllll}
F & 0_{m \times m} & 0_{m \times m} & \ldots & 0_{m \times m} \\
D C & F & 0_{m \times m} & \ldots & 0_{m \times m} \\
D A C & D C & F & \ldots & 0_{m \times m} \\
\vdots & \vdots & \vdots & \ddots & \vdots \\
D A^{i-2} C & D A^{i-3} C & D A^{i-4} C & \ldots & F
\end{array}\right] \in \mathbb{R}^{i m \times i m}
$$

\subsection{Hankel matrix notation}

Hankel matrices are frequently used in realization theory and subspace system identification. The special structure of a Hankel matrix as well as some matching notations, which are frequently used througout, are defined in the following.

\section{Definition 2.2. (Hankel matrix)}

Given a (vector or matrix) sequence of data

$$
s_{t} \in \mathbb{R}^{n r \times n s} \forall t=0,1,2, \ldots, t_{0}, t_{0}+1, \ldots,
$$

where $n r$ is the number of rows in $s_{t}$ and $n c$ is the number of columns in $s_{t}$.

Define integer numbers $t_{0}, L$ and $K$ and define the matrix $S_{t}$ as follows

$$
S_{t_{0} \mid L} \stackrel{\text { def }}{=}\left[\begin{array}{lllll}
s_{t_{0}} & s_{t_{0}+1} & s_{t_{0}+2} & \ldots & s_{t_{0}+K-1} \\
s_{t_{0}+1} & s_{t_{0}+2} & s_{t_{0}+3} & \ldots & s_{t_{0}+K} \\
\vdots & \vdots & \vdots & \ddots & \vdots \\
s_{t_{0}+L-1} & s_{t_{0}+L} & s_{t_{0}+L+1} & \ldots & s_{t_{0}+L+K-2}
\end{array}\right] \in \mathbb{R}^{L n r \times K n c}
$$

which is defined as a Hankel matrix because of the special structure. The integer numbers $t_{0}, L$ and $K$ are defined as follows:

- $t_{0}$ start index or initial time in the sequence, $s_{t_{0}}$, which is the upper left block in the Hankel matrix.

- $L$ is the number of $n r$-block rows in $S_{t_{0} \mid L}$.

- $K$ is the number of $n c$-block columns in $S_{t_{0} \mid L}$. 
A Hankel matrix is symmetric and the elements are constant across the anti-diagonals. We are usually working with vector sequences in subspace system identification, i.e., $s_{t}$ is a vector in this case and hence, $n c=1$. Examples of such vector processes, to be used in the above Hankel-matrix definition, are the measured process outputs, $y_{t} \in \mathbb{R}^{m}$, and possibly known inputs, $u_{t} \in \mathbb{R}^{r}$. Also define

$$
y_{j \mid i} \stackrel{\text { def }}{=}\left[\begin{array}{llll}
y_{j}^{T} & y_{j+1}^{T} & \cdots & y_{j+i-1}^{T}
\end{array}\right]^{T} \in \mathbb{R}^{i m}
$$

which is refereed to as an extended (output) vector, for later use.

\subsection{Projections}

Given two matrices $A \in \mathbb{R}^{i \times k}$ and $B \in \mathbb{R}^{j \times k}$. The orthogonal projection of the row space of $A$ onto the row space of $B$ is defined as

$$
A / B=A B^{T}\left(B B^{T}\right)^{\dagger} B
$$

The orthogonal projection of the row space of $A$ onto the orthogonal complement of the row space of $B$ is defined as

$$
A B^{\perp}=A-A / B=A-A B^{T}\left(B B^{T}\right)^{\dagger} B
$$

The following properties are frequently used

$$
\begin{gathered}
A /\left[\begin{array}{l}
A \\
B
\end{array}\right]=A \\
A /\left[\begin{array}{l}
A \\
B
\end{array}\right]^{\perp}=0
\end{gathered}
$$

Prof of equations (13) and (14) can be found in e.g., Di Ruscio (1997b). The MoorePenrose pseudo-inverse of a matrix $A \in \mathbb{R}^{i \times k}$ where $k>i$ is defined as $A^{\dagger}=A^{T}\left(A A^{T}\right)^{-1}$. Furthermore, consistent with equation (12) we will use the definition

$$
B^{\perp}=I_{k}-B^{T}\left(B B^{T}\right)^{\dagger} B
$$

throughout the paper. Note also the properties that $\left(B^{\perp}\right)^{T}=B^{\perp}$ and $B^{\perp} B^{\perp}=B^{\perp}$.

\section{Subspace System Identification}

\subsection{Subspace identification of the states}

Consider a discrete time Kalman filter on innovations form, i.e.,

$$
\begin{gathered}
\bar{x}_{k+1}=A \bar{x}_{k}+B u_{k}+K \varepsilon_{k} \\
y_{k}=D \bar{x}_{k}+E u_{k}+\varepsilon_{k}
\end{gathered}
$$

where $\bar{x}_{k} \in \mathbb{R}^{n}$ is the predicted state in a minimum variance sense, $\varepsilon_{k} \in \mathbb{R}^{m}$ is the innovations at discrete time $k$, i.e., the part of $\mathrm{y}_{k} \in \mathbb{P}^{m}$ that cannot be predicted from past data (i.e. known past inputs and outputs) and the present input. Furthermore, $\bar{y}_{k}=D \bar{x}_{k}+E u_{k}$ is the prediction of $y_{k}$, and $\varepsilon_{k}$ is white noise with covariance matrix $\Delta=E\left(\varepsilon_{k} \varepsilon_{k}^{T}\right)$. Here $\varepsilon_{k}=F e_{k}$ is the innovations and the models (1) and (2) are therefore 
equivalent with the Kalman filter equations (16) and (17). Furthermore, we have that $K=C F^{-1}$ and $\Delta=E\left(\varepsilon_{k} \varepsilon_{k}^{T}\right)=F F^{T}$, when $F$ is non-singular, i.e., when the system is not deterministic and when the Kalman filter exists.

A well known belief is that the states is a function of the past. Let us have a lock at this statement. The predicted state at time $k:=t_{0}+J$, i.e. $\bar{x}_{t_{0}+J}$ of a Kalman filter with the initial predicted state at $k:=t_{0}$, i.e. $\bar{x}_{t_{0}}$ given, can be expressed as

$$
\bar{x}_{t_{0}+J}=\tilde{C}_{J}^{s} y_{t_{0} \mid J}+\tilde{C}_{J}^{d} u_{t_{0} \mid J}+(A-K D)^{J} \bar{x}_{t_{0}}
$$

where $\tilde{C}_{J}^{s}=C_{J}(A-K D, K)$ is the reversed extended controllability matrix of the pair $(A-K D, K), \tilde{C}_{J}^{d}=C_{J}(A-K D, B-K E)$ is the reversed extended controllability matrix of the pair $(A-K D, B-K E)$ and $\bar{x}_{t_{0}}$ is the initial predicted state (estimate) at the initial discrete time $t_{0}$. See equation (5) for the definition of the reversed controllability matrix. $J$ is the past horizon, i.e., the number of past outputs and inputs used to define the predicted state (estimate) $\bar{x}_{t_{0}+J}$ at the discrete time $t_{0}+J$.

Using equation (18) for different $t_{0}$, i.e. for $t_{0}, t_{0}+1, t_{0}+2, \ldots, t_{0}+K-1$, gives the matrix equation

$$
X_{t_{0}+J}=\tilde{C}_{J}^{s} Y_{t_{0} \mid J}+\tilde{C}_{J}^{d} U_{t_{0} \mid J}+(A-K D)^{J} X_{t_{0}}
$$

where

$$
\begin{aligned}
X_{t_{0}+J} & =\left[\begin{array}{lllll}
\bar{x}_{t_{0}+J} & \bar{x}_{t_{0}+J+1} & \ldots & \bar{x}_{t_{0}+J+K-1}
\end{array}\right] \in \mathbb{R}^{n \times K} \\
X_{t_{0}} & =\left[\begin{array}{lllll}
\bar{x}_{t_{0}} & \bar{x}_{t_{0}+1} & \ldots & \bar{x}_{t_{0}+K-1}
\end{array}\right] \in \mathbb{R}^{n \times K}
\end{aligned}
$$

where $K$ is the number of columns in the data matrices. Note that $K$ also is equal to the number of vector equations of the form equation (18) which is used to form the matrix version equation (19). Note also that the state matrix $X_{t_{0}}$ can be eliminated from equation (19) by using the relationship

$$
Y_{t_{0} \mid J}=O_{J} X_{t_{0}}+H_{J}^{d} U_{t_{0} \mid J+g-1}+H_{J}^{s} E_{t_{0} \mid J}
$$

which we have deduced from the innovations form, state space model (1) and (2). Putting $t_{0}=: t_{0} \mid J$ in equation (22) gives

$$
Y_{t_{0}+J \mid L}=O_{L} X_{t_{0}+J}+H_{L}^{d} U_{t_{0}+J \mid L+g-1}+H_{L}^{s} E_{t_{0}+J \mid L}
$$

Using equation (19) to eliminate $X_{t_{0}+J}$ in equation (23) gives a matrix equation which relates the future data matrices $Y_{t_{0}+J \mid L}, U_{t_{0}+J \mid L+g-1}, E_{t_{0}+J \mid L}$ and the past data matrices $Y_{t_{0} \mid J}, U_{t_{0} \mid J}, E_{t_{0} \mid J}$.

The data is usually defined at time instant (or number of observations) $k=1,2, \ldots, N$. Hence, $t_{0}=1$ in this case. However, we are often defining $t_{0}=0$ which corresponds to data defined at $k=0,1, \ldots, N-1$. The bar used to indicate predicted state is often omitted. Hence, for simplicity of notation, we define the following from equations (19), (22) and (23),

$$
\begin{gathered}
Y_{0 \mid J}=O_{J} X_{0}+H_{J}^{d} U_{0 \mid J+g-1}+H_{J}^{s} E_{0 \mid J} \\
X_{J}=\tilde{C}_{J}^{s} Y_{0 \mid J}+\tilde{C}_{J}^{d} U_{0 \mid J}+(A-K D)^{J} X_{0} \\
Y_{I \mid I}=O_{L} X_{I}+H_{I}^{d} U_{J \mid I+g-1}+H_{L}^{s} E_{J \mid L}
\end{gathered}
$$


for lather use. Furthermore, equations (26) and (25) gives

$$
Y_{J \mid L}=\left[\begin{array}{lll}
H_{L}^{d} & O_{L} \tilde{C}_{J}^{d} & O_{L} \tilde{C}_{J}^{s}
\end{array}\right]\left[\begin{array}{l}
U_{J \mid L+g-1} \\
U_{0 \mid J} \\
Y_{0 \mid J}
\end{array}\right]+O_{L}(A-K D)^{J} X_{0}+H_{L}^{s} E_{J \mid L}
$$

Equation (27) is important for understanding a SID algorithm, because, it gives the relationship between the past and the future. Note also the terms in equation (27) which are 'proportional' with the extended observability matrix $O_{L}$. From equation (27) we see that the effect from the future inputs, $U_{J \mid L+g-1}$, and the future noise, $E_{J \mid L}$, have to be removed from the future outputs, $Y_{J \mid L}$, in order to recover the subspace spanned by the extended observability matrix, $O_{L}$. A variation of this equation, in which the term $X_{0}$ is eliminated by using equations (22) or (24) is presented in Di Ruscio (1997b). Note also that equations (25) and (24) gives

$$
\begin{gathered}
X_{J}=\left[\begin{array}{cc}
P_{J}^{u} & P_{J}^{y}
\end{array}\right]\left[\begin{array}{c}
U_{0 \mid J} \\
Y_{0 \mid J}
\end{array}\right]-P_{J}^{e} E_{0 \mid J} \\
P_{J}^{u}=\tilde{C}_{J}^{d}-(A-K D)^{J} O_{J}^{\dagger} H_{J}^{d} \\
P_{J}^{y}=\tilde{C}_{J}^{s}+(A-K D)^{J} O_{J}^{\dagger} \\
P_{J}^{e}=(A-K D)^{J} O_{J}^{\dagger} H_{J}^{s}
\end{gathered}
$$

where we for the sake of simplicity and without loss of generality have put $g=1$. Equation (28) is useful because it shows that the future states $X_{J}$ is in the range of a matrix consisting of past inputs, $U_{0 \mid J}$, and past outputs, $Y_{0 \mid J}$ (in the deterministic case or when $J \rightarrow \infty)$. Note that we have introduced the notation, $P_{J}^{u}$, in order to represent the influence from the past inputs upon the future. Combining equations (28) and (26) gives an alternative to equation (27), i.e. the 'past-future' matrix equation,

$$
Y_{J \mid L}=\left[\begin{array}{lll}
H_{L}^{d} & O_{L} P_{J}^{u} & O_{L} P_{J}^{y}
\end{array}\right]\left[\begin{array}{l}
U_{J \mid L+g-1} \\
U_{0 \mid J} \\
Y_{0 \mid J}
\end{array}\right]-O_{L} P_{J}^{e} E_{0 \mid J}+H_{L}^{s} E_{J \mid L}
$$

The two last terms in equation (32) cannot be predicted from data, i.e., because $E_{0 \mid J}$ and $E_{J \mid L}$ are built from the innovations process $e_{k}$.

It is important to note that a consistent estimate of the system dynamics can be obtained by choosing $L$ and $N$ properly. Choosing $L_{\min } \leqslant L$ where $L_{\min }=$ $n+\operatorname{rank}(D)-1$ and letting $N \rightarrow \infty$, is in general, necessary conditions for a consistent estimate of the dynamics. See Section 3.2 for further details.

On the other side, it is in general, also necessary to let $J \rightarrow \infty$ in order to obtain a consistent estimate of the states. The reason for this is that the term $(A-K D)^{J}=0$ in this case. Hence, the effect of the initial state matrix $X_{0}$ on the future states $X_{J}$ has died out. We have the following Lemma. 
Lemma 3.1. (Subspace identification of the states)

Let $K \rightarrow \infty$ in the data matrices. The projected state matrix is defined as

$$
\begin{aligned}
X_{J} /\left[\begin{array}{l}
U_{J \mid L+g-1} \\
U_{0 \mid J} \\
Y_{0 \mid J}
\end{array}\right] & =O_{L}^{t}(\overbrace{Y_{J \mid L} /\left[\begin{array}{l}
U_{J \mid L+g-1} \\
U_{0 \mid J} \\
Y_{0 \mid J}
\end{array}\right]}^{Z_{J \mid L}^{d}}-H_{L}^{d} U_{J \mid L+g-1}) \\
& =\tilde{C}_{J}^{s} Y_{0 \mid J}+\tilde{C}_{J}^{d} U_{0 \mid J}+(A-K D)^{J} X_{\mathrm{o}} /\left[\begin{array}{l}
U_{J \mid L+g-1} \\
U_{0 \mid J} \\
Y_{0 \mid J}
\end{array}\right]
\end{aligned}
$$

Consider the case when

$$
(A-K D)^{J} X_{0} /\left[\begin{array}{l}
U_{J \mid L+g-1} \\
U_{0 \mid J} \\
Y_{0 \mid J}
\end{array}\right]=0
$$

which is satisfied when $J \rightarrow \infty$ and $(A-K D)$ is stable. This gives

$$
X_{J} /\left[\begin{array}{l}
U_{J \mid L+g-1} \\
U_{0 \mid J} \\
Y_{0 \mid J}
\end{array}\right]=X_{J}
$$

and hence we have, in general, the following expression for the future states

$$
X_{J}=O_{L}^{ \pm}(\overbrace{Y_{J \mid L} /\left[\begin{array}{l}
U_{J \mid L+g-1} \\
U_{0 \mid J} \\
Y_{0 \mid J}
\end{array}\right]}^{Z_{J \mid L}^{d}}-H_{L}^{d} U_{J \mid L+g-1})
$$

Proof 3.1. The proof is divided into two parts.

Part 1. The relationship between the future data matrices is given by

$$
Y_{J \mid L}=O_{L} X_{J}+H_{L}^{d} U_{J \mid L+g-1}+H_{L}^{s} E_{J \mid L}
$$

Projecting the row space of each term in equation (37) onto the row space of

$$
\left[\begin{array}{l}
\mathrm{U}_{J \mid L+g-1} \\
U_{0 \mid J} \\
Y_{0 \mid J}
\end{array}\right]
$$


gives

$$
Y_{J \mid L} /\left[\begin{array}{l}
U_{J \mid L+g-1} \\
U_{0 \mid J} \\
Y_{0 \mid J}
\end{array}\right]=O_{L} X_{J} /\left[\begin{array}{l}
U_{J \mid L+g-1} \\
U_{0 \mid J} \\
Y_{0 \mid J}
\end{array}\right]+H_{L}^{d} U_{J \mid L+g-1}+d E_{1}
$$

where the error term is given by

$$
d E_{1}=H_{L}^{s} E_{J \mid L} /\left[\begin{array}{l}
U_{J \mid L+g-1} \\
U_{0 \mid J} \\
Y_{0 \mid J}
\end{array}\right]
$$

It make sense to assume that future noise matrix $E_{J \mid L}$ is uncorrelated with past data and the future inputs, hence, we have that (w.p.1)

$$
\lim _{K \rightarrow \infty} d E_{1}=0
$$

Part 2. Equation (25) gives the relationship between the future state matrix $X_{J}$ and the past data matrices. Projecting the row space of each term in this equation onto the row space of

$$
\left[\begin{array}{l}
U_{J \mid L+g-1} \\
U_{0 \mid J} \\
Y_{0 \mid J}
\end{array}\right]
$$

gives

$$
X_{J} /\left[\begin{array}{l}
U_{J \mid L+g-1} \\
U_{0 \mid J} \\
Y_{0 \mid J}
\end{array}\right]=\tilde{C}_{J}^{s} Y_{0 \mid J}+\tilde{C}_{J}^{d} U_{0 \mid J}+(A-K D)^{J} X_{0} /\left[\begin{array}{l}
U_{J \mid L+g-1} \\
U_{0 \mid J} \\
Y_{0 \mid J}
\end{array}\right]
$$

Letting $J \rightarrow \infty$ (or assuming the last term to be zero) gives

$$
X_{J} /\left[\begin{array}{l}
U_{J \mid L+g-1} \\
U_{0 \mid J} \\
Y_{0 \mid J}
\end{array}\right]=\tilde{C}_{J}^{s} Y_{0 \mid J}+\tilde{C}_{J}^{d} U_{0 \mid J}
$$

Letting $J \rightarrow \infty$ and assuming the system matrix $(A-K D)$ for the predicted outputs to be stable in equation (25) shows that

$$
X_{J}=\tilde{C}_{J}^{s} Y_{0 \mid J}+\tilde{C}_{J}^{d} U_{0 \mid J}
$$

Comparing equations (42) and (43) gives

$$
X_{J}=X_{J} /\left[\begin{array}{l}
U_{J \mid L+g-1} \\
U_{0 \mid J} \\
Y_{0 \mid J}
\end{array}\right]
$$

Using equation (44) in equation (38) and solving for $X_{J}$ gives equations (36). 
The condition in equation (35) is usually satisfied for large $J$, i.e., we have that $\lim _{J \rightarrow \infty}(A-K D)^{J}=0$ when $A-K D$ is stable. Note also that the eigenvalues of $A-K D$ usually are close to zero for 'large' process noise (or 'small' measurements noise). Then, $(A-K D)^{J}$ is approximately zero even for relatively small numbers $J$. We will now discuss some special cases

\section{Lemma 3.2. (SID of states: white input)}

Consider a combined deterministic and stochastic system excited with a white input signal. Then

$$
X_{J}=O_{L}^{\dagger} Y_{J \mid L} /\left[\begin{array}{l}
U_{J \mid L+g-1} \\
U_{0 \mid J} \\
Y_{0 \mid J}
\end{array}\right] U_{J \mid L+g-1}^{J}
$$

when $J \rightarrow \infty$.

Proof 3.2. This result follows from the proof of Lemma 3.1 and equation (36) and using that

$$
X_{J} U_{J \mid L+g-1}^{\perp}=X_{J}
$$

when $u_{k}$ is white and, hence, $X_{0} / U_{J \mid L+g-1}=0$.

Lemma 3.3. (SID of states: pure stochastic system)

Consider a stochastic system. Then we simply have that

$$
X_{J}=O_{L}^{\dagger} Y_{J \mid L} / Y_{0 \mid J}
$$

when $J \rightarrow \infty$ or when $(A-K D)^{J} X_{0} / Y_{0 \mid J}=0$ is satisfied.

Proof 3.3. This result follows from the proof of Lemma 3.1 by putting the measured input variables equal to zero.

Lemma 3.1 shows that it is in general (i.e. for colored input signals) necessary to know the deterministic part of the system, i.e., the Toepliz matrix $H_{L}^{d}$ in equation (36), in order to properly identify the states. This means that the matrices $B$ and $E$ in addition to $D$ and $A$ has to be identified prior to computing the states. I.e. we need to know the deterministic part of the model. However, a special case is given by Lemma 3.2 and equation (45) which shows that the states can be identified directly when the input signals is white. Note also that the extended observability matrix $O_{L}$ is needed in equations (36) and (45). $O_{L}$ can be identified directly from the data. This is proved in the next Section 3.2, and this is indeed the natural step in a SID algorithm.

In the case of a white input signal or when $J \rightarrow \infty$ then, $H_{L}^{d}$, and the state matrix, $X_{J}$, can be computed as by the N4SID algorithm, Van Overschee and De Moor (1996). From equations (28) and (32) we have the following lemma

Lemma 3.4. (States, $X_{J}$, and Toepliz matrix $H_{L}^{d}$ : N4SID)

The following LS solution

$$
\left[\begin{array}{lll}
H_{L}^{d} & O_{L} P_{J}^{u} & O_{L} P_{J}^{y}
\end{array}\right]=Y_{J \mid L}\left[\begin{array}{l}
U_{J \mid L+g-1} \\
U_{0 \mid J} \\
Y_{0 \mid J}
\end{array}\right]^{\dagger}+d E
$$


holds in:

(i) The deterministic case, provided the input is PE of order $J+L+g-1$. The error term, $d E=0$, in this case.

(ii) When $J \rightarrow \infty$, and the input is PE of infinite order. The error term, $d E=0$, in this case.

(iii) A white $u_{k}$ gives a consistent estimate of $H_{L}^{d}$ irrespective of $J>0$. However, $O_{L} P_{J}^{u}$ and $O_{L} P_{J}^{y}$ are not consistent estimates in this case. The first $m L \times(L+g) r$ part of the error term, $d E$, is zero in this case.

Hence, under conditions (i) and (ii), $O_{L} P_{J}^{u}$ and $O_{L} P_{J}^{y}$ can be computed as in equation (48). Then the states can be consistently estimated as

$$
X_{J}=O_{L}^{\dagger}\left[O_{L} P_{J}^{u} \quad O_{L} P_{J}^{y}\right]\left[\begin{array}{c}
U_{0 \mid J} \\
Y_{0 \mid J}
\end{array}\right]
$$

provided conditions (i) and (ii) are satisfied, and $O_{L}^{\dagger}$ is known.

Proof 3.4. The PE conditions in the lemma are due to the existence of the LS solution, i.e., the concatenated matrix

$$
\left[\begin{array}{l}
U_{J \mid L+g-1} \\
U_{0 \mid J}
\end{array}\right]
$$

has to be of full row rank. From equation (32) we have that the error term in the LS problem is

$$
d E=\left(-O_{L} P_{J}^{e} E_{0 \mid J}+H_{L}^{s} E_{J \mid L}\right)\left[\begin{array}{l}
U_{J \mid L+g-1} \\
U_{0 \mid J} \\
Y_{0 \mid J}
\end{array}\right]^{\dagger}=-O_{L} P_{J}^{e} E_{0 \mid J}\left[\begin{array}{l}
U_{J \mid L+g-1} \\
U_{0 \mid J} \\
Y_{0 \mid J}
\end{array}\right]^{\dagger}
$$

It is clear from equation (31) that the error term $d E=0$ when $J \rightarrow \infty$. This proves condition (i) in the lemma. Furthermore, the error term, $d E=0$, in the deterministic case because $E_{0 \mid J}=0$ in this case. This proves condition (ii). Analyzing the error term, $d E$, for a white input shows that the error term is of the form

$$
d E=\left[\begin{array}{lll}
0_{m L \times(L+g) r} & d E_{2} & d E_{3}
\end{array}\right]^{\dagger}
$$

where the $d E_{2}$ and $d E_{3}$ are submatrices in $d E$ different from zero. Note that $d E_{2}=0$ for strictly proper systems, $g=0$, when $u_{k}$ is white. This proves condition (iii).

The states can then be computed by using equations (28) or (43), provided conditions (i) or (ii) are satisfied.

One should note that in the N4SID algorithm the past horizon is put equal to the future horizon (N4SID parameter $(i)$ ). In order for the above lemma to give the same results as in the N4SID algorithm we have to put $i=L+1, J=L+1$ and $g=1$, i.e so that $J+L=2 L+1=2 i$. Note that this last result does not hold in general. It holds in the deterministic case or when $J \rightarrow \infty$. The extended observability matrix $O_{L}$ can be computed as presented in the next section.

\subsection{The extended observability matrix}

An important first step in the SID algorithm is the identification of the system order, $n$, and the extended observability matrix $O_{L+1}$. The reason for searching for 
$O_{L+1}$ is that we have to define $A$ from the shift invariance property, Kung (1978), or a similar method, e.g. as in Di Ruscio (1995). The key is to compute a special projection matrix from the known data. This is done without using the states. We will in this section show how this can be done for coloured input signals.

Lemma 3.5. (SID of the extended observability matrix)

The following projections are equivalent

$$
\begin{gathered}
Z_{J \mid L+1}=\left(Y_{J \mid L+1} /\left[\begin{array}{c}
U_{J \mid L+g} \\
U_{0 \mid J} \\
Y_{0 \mid J}
\end{array}\right]\right) U_{J \mid L+g}^{\perp} \\
Z_{J \mid L+1}=\left(Y_{J \mid L+1} U_{J \mid L+g}^{\perp}\right) /\left(\left[\begin{array}{l}
U_{0 \mid J} \\
Y_{0 \mid J}
\end{array}\right] U_{J \mid L+g}^{\perp}\right) \\
Z_{J \mid L+1}=Y_{J \mid L+1} /\left(\left[\begin{array}{c}
U_{0 \mid J} \\
Y_{0 \mid J}
\end{array}\right] U_{J \mid L+g}^{\perp}\right)
\end{gathered}
$$

Furthermore, $Z_{J \mid L+1}$ is related to the extended observability matrix $O_{L+1}$ as

$$
Z_{J \mid L+1}=O_{L+1} X_{J}^{a}
$$

where the 'projected states' $X_{J}^{a}$ can be expressed as

$$
\begin{aligned}
X_{J}^{a} & =\left(X_{J} /\left[\begin{array}{l}
U_{J \mid L+g} \\
U_{0 \mid J} \\
Y_{0 \mid J}
\end{array}\right]\right) U_{J \mid L+g}^{\perp} \\
& =\left(\tilde{C}_{J}^{d} U_{0 \mid J}+\tilde{C}_{J}^{s} Y_{0 \mid J}-(A-K D)^{J} X_{0} /\left[\begin{array}{c}
U_{J \mid L+g} \\
U_{0 \mid J} \\
Y_{0 \mid J}
\end{array}\right]\right) U_{J \mid L+g}^{\perp} \\
& =\left(X_{J}-(A-K D)^{J} X_{0}\left[\begin{array}{l}
U_{J \mid L+g} \\
U_{0 \mid J} \\
Y_{0 \mid J}
\end{array}\right]^{\perp}\right) U_{J \mid L+g}^{\perp} \\
& =\left(X_{J}+(A-K D)^{J} O_{J}^{\dagger} H_{J}^{s} E_{0 \mid J}\left[\begin{array}{l}
U_{J \mid L+g} \\
U_{0 \mid J} \\
Y_{0 \mid J}
\end{array}\right]^{\perp}\right) U_{J \mid L+g}^{\perp}
\end{aligned}
$$

Furthermore, the column space of $Z_{J \mid L+1}$ coincide with the column space of $O_{L+1}$ and $n=\operatorname{rank}\left(Z_{J \mid L+1}\right)$ if $\operatorname{rank}\left(X_{J}^{a}\right)=n$.

Proof 3.5. The proof is divided into two parts. In the first part of equations (52) and (55) with the alternative expressions in equations (56) to (58) are proved. In the second part the equivalence with equations (52), (53) and (54) are proved. 
Part 1. Projecting the row space of each term in equation (26) with $L:=L+1$ onto the row space of

$$
\left[\begin{array}{l}
U_{J \mid L+g-1} \\
U_{0 \mid J} \\
Y_{0 \mid J}
\end{array}\right]
$$

gives

$$
Y_{J \mid L+1} /\left[\begin{array}{l}
U_{J \mid L+g-1} \\
U_{0 \mid J} \\
Y_{0 \mid J}
\end{array}\right]=O_{L+1} X_{J} /\left[\begin{array}{l}
U_{J \mid L+g-1} \\
U_{0 \mid J} \\
Y_{0 \mid J}
\end{array}\right]+H_{L+1}^{d} U_{J \mid L+g-1}+d E_{1}
$$

where we have used equation (13). Then, w.p.1

$$
\lim _{K \rightarrow \infty} d E_{1}=0
$$

where the error term, $d E_{1}$, is given by equation (39) with $L:=L+1$. Removing the effect of the future input matrix, $U_{J \mid L+g-1}$, on equation (60) gives equations (52) and (55) with $X_{J}^{a}$ as in equation (56).

Furthermore, projecting the row space of each term in equation (25) onto the row space of

$$
\left[\begin{array}{l}
U_{J \mid L+g-1} \\
U_{0 \mid J} \\
Y_{0 \mid J}
\end{array}\right]
$$

gives

$$
X_{J} /\left[\begin{array}{l}
U_{J \mid L+g-1} \\
U_{0 \mid J} \\
Y_{0 \mid J}
\end{array}\right]=\tilde{C}_{J}^{s} Y_{0 \mid J}+\tilde{C}_{J}^{d} U_{0 \mid J}+(A-K D)^{J} X_{0} /\left[\begin{array}{l}
U_{J \mid L+g-1} \\
U_{0 \mid J} \\
Y_{0 \mid J}
\end{array}\right]
$$

From equation (25) we have that

$$
\tilde{C}_{J}^{s} Y_{0 \mid J}+\tilde{C}_{J}^{d} U_{0 \mid J}=X_{J}-(A-K D)^{J} X_{0}
$$

Combining equations (60), (62) and (63) gives equations (52) and (57)-(58).

Part 2. It is proved in Di Ruscio (1997) that

$$
\begin{aligned}
Z_{J \mid L+1} & =Y_{J \mid L+1} /\left[\begin{array}{l}
U_{J \mid L+g} \\
W
\end{array}\right] U_{J \mid L+g}^{\perp} \\
& =Y_{J \mid L+1} U_{J \mid L+g}^{\perp} W^{T}\left(W U_{J \mid L+g}^{\perp} W^{T}\right)^{-1} W U_{J \mid L+g}^{\perp}
\end{aligned}
$$

where

$$
W=\left[\begin{array}{l}
U_{0 \mid J} \\
Y_{0 \mid J}
\end{array}\right]
$$

Using that $U_{J \mid L+g}^{\perp} U_{J \mid L+g}^{\perp}=U_{J \mid L+g}^{\perp}$ in equation (64) proves the equivalence between equations (53), (54) and (52). 
Lemma 3.6. (Consistency: Stochastic and deterministic systems)

Let $J \rightarrow \infty$, then

$$
Z_{J \mid L+1}=O_{L+1} X_{J} U_{J \mid L+g}^{1}
$$

where $Z_{J \mid L+1}$ is defined as in Lemma 3.5. A sufficient condition for consistency, and that $O_{L+1}$ is contained in the column space of $Z_{J \mid L+1}$, is that there are no pure state feedback.

Proof 3.6. Letting $J \rightarrow \infty$ in equation (58) gives (66). This can also be proved by using equation (44) in equation (56). Furthermore, if there are pure state feedback then $X_{J} U_{J \mid L+g}^{\perp}$ will lose rank below the normal rank which is $n$.

\section{Lemma 3.7. (Deterministic systems)}

For pure deterministic systems we have that equation (66) can be changed to

$$
Z_{J \mid L+1}=: Y_{J \mid L+1} U_{J \mid L+g}^{\perp}=O_{L+1} X_{J} U_{J \mid L+g}^{\perp}
$$

The extended observability matrix $O_{L+1}$ can be computed from the column space of $Y_{J \mid L+1} U_{J \mid L+g}^{\perp}$. Furthermore, one can let $J=0$ in the deterministic case.

Proof 3.7. This follows from equation (66) and Lemma 3.5 by excluding the projection which removes the noise.

\section{Lemma 3.8. (Stochastic systems)}

For pure stochastic systems we have that equation (66) can be changed to

$$
Z_{J \mid L+1}=: Y_{J \mid L+1} / Y_{0 \mid J}=O_{L+1} X_{J}
$$

The extended observability matrix $O_{L+1}$ can be computed from the column space of $Y_{J \mid L+1} / Y_{0 \mid J}$

Proof 3.8. This follows from equation (66) and Lemma 3.5 by excluding the input matrices from the equations and definitions.

\subsection{Identification of the stochastic subsystem}

We will in this section prove that, when the extended observabilit matrix is known (from Section 3.2), the kalman filter gain matrix and the noise innovations process can be identified directly from the data.

\section{Lemma 3.9. (The innovations)}

Define the following projection from the data

$$
Z_{J \mid L+1}^{s}=Y_{J \mid L+1}-Y_{J \mid L+1} /\left[\begin{array}{l}
U_{J \mid L+g} \\
U_{0 \mid J} \\
Y_{0 \mid J}
\end{array}\right]=Y_{J \mid L+1}\left[\begin{array}{l}
U_{J \mid L+g} \\
U_{0 \mid J} \\
Y_{0 \mid J}
\end{array}\right]^{\perp}
$$

Then w.p.1 as $J \rightarrow \infty$

$$
Z_{J \mid L+1}^{s}=H_{L+1}^{s} E_{J \mid L+1}
$$


Hence, the Toeplitz matrix $H_{L+1}^{s}$ (with Markov matrices $F, D C, \ldots, D A^{L-1} C$ ) for the stochastic subsystem is in the column space of

$$
\frac{1}{\sqrt{K}} Z_{J \mid L+1}^{s}
$$

since

$$
\frac{1}{K} E_{J \mid L+1} E_{J \mid L+1}^{T}=I_{L+1 \times L+1}
$$

Proof 3.9. The relationship between the future data matrices is given by

$$
Y_{J \mid L}=O_{L} X_{J}+H_{L}^{d} U_{J \mid L+g-1}+H_{L}^{s} E_{J \mid L}
$$

Projecting the row space of each term in equation (71) onto the row space of

$$
\left[\begin{array}{l}
U_{J \mid L+g-1} \\
U_{0 \mid J} \\
Y_{0 \mid J}
\end{array}\right]
$$

gives

$$
Y_{J \mid L} /\left[\begin{array}{l}
U_{J \mid L+g-1} \\
U_{0 \mid J} \\
Y_{0 \mid J}
\end{array}\right]=O_{L} X_{J} /\left[\begin{array}{l}
U_{J \mid L+g-1} \\
U_{0 \mid J} \\
Y_{0 \mid J}
\end{array}\right]+H_{L}^{d} U_{J \mid L+g-1}+d E_{1}
$$

then, w.p.1

$$
\lim _{K \rightarrow \infty} d E_{1}=0
$$

where $d E_{1}$ is given in equation (39). Furthermore,

$$
\lim _{J \rightarrow \infty} X_{J} /\left[\begin{array}{l}
U_{J \mid L+g-1} \\
U_{0 \mid J} \\
Y_{0 \mid J}
\end{array}\right]=X_{J}
$$

where we have used equations (44) and (38). From equations (71), (72) and (74) we have that

$$
Y_{J \mid L}-Y_{J \mid L} /\left[\begin{array}{l}
U_{J \mid L+g-1} \\
U_{0 \mid J} \\
Y_{0 \mid J}
\end{array}\right]=H_{L}^{s} E_{J \mid L}
$$

Putting $L:=L+1$ in equation (75) gives equation (69).

It is now possible to directly identify the matrices $C$ and $F$ in the innovations model equations (1) and (2) and $K$ and $\Delta$ in the Kalman filter equations (16) and (17). Two methods are presented in the following. The first one is a direct covariance based method for computing $K$ and $\Delta$ and the second one is a more numerically reliable 'square root' based method for computing $C$ and $F$. 


\section{Lemma 3.10. (Correlation method for $K$ and $\Delta$ )}

Define the projection matrix $Z_{J \mid L+1}^{s}$ as in equation (69) and define the correlation matrix

$$
\Delta_{L+1}=\frac{1}{K} Z_{J \mid L+1}^{s}\left(Z_{J \mid L+1}^{s}\right)^{T}=H_{L+1}^{s}\left(H_{L+1}^{s}\right)^{T}
$$

where the Toepliz matrix $H_{L+1}^{s}$ can be partitioned as

$$
H_{L+1}^{s}=\left[\begin{array}{ll}
F & 0_{m \times L m} \\
O_{L} C & H_{L}^{s}
\end{array}\right]
$$

where $C=K F$. Hence, equation (76) can be written as

$$
\Delta_{L+1}=\left[\begin{array}{ll}
\Delta_{11} & \Delta_{12} \\
\Delta_{21} & \Delta_{22}
\end{array}\right]=\left[\begin{array}{ll}
F F^{T} & F\left(O_{L} C\right)^{T} \\
O_{L} C F^{T} & O_{L} C\left(O_{L} C\right)^{T}+H_{L}^{s}\left(H_{L}^{s}\right)^{T}
\end{array}\right]
$$

From this we have

$$
E\left(\varepsilon_{k} \varepsilon_{k}^{T}\right)=F F^{T}=\Delta_{11}
$$

and

$$
K=C F^{-1}=O_{L}^{\dagger} \Delta_{21} \Delta_{11}^{-1}
$$

\section{Lemma 3.11. (Square-root method for $C$ and $F$ )}

The LQ decomposition of

$$
\frac{1}{\sqrt{K}} Z_{J \mid L+1}^{s}
$$

gives

$$
\frac{1}{\sqrt{K}} Z_{J \mid L+1}^{s}=R_{33} Q_{3}
$$

Then, the Toeplitz matrix $H_{L+1}^{s}$, and the Markov matrices $F, D C, \ldots, D A^{L-1} C$, are given directly by

$$
H_{L+1}^{s}=R_{33}
$$

$F$ can be taken as one of the diagonal $m \times m$ block matrices in $R_{33}$, e.g. the lower left sub-matrix, i.e.

$$
F=R_{33}(L m+1:(L+1) m, L m+1:(L+1) m)
$$

Furthermore,

$$
O_{L} C=R_{33}(m+1:(L+1) m, 1: m)
$$

The system matrix $C$ is given by

$$
C=O_{L}^{\dagger} O_{L} C=O_{L}^{\dagger} R_{33}(m+1:(L+1) m, 1: m)
$$

The Kalman filter gain matrix and the innovations covariance matrix are given by

$$
\begin{aligned}
K & =C F^{-1} \\
& =O_{L}^{\dagger} R_{33}(m+1:(L+1) m, 1: m) R_{33}^{-1}(L m+1:(L+1) m, L m+1:(L+1) m) \\
\Delta & =F F^{T}
\end{aligned}
$$




\subsection{SID of the deterministic subsystem}

The parameters in the $B$ and $E$ matrices can be computed from an optimal least squares problem. A solution to this is given in the following

\section{Lemma 3.12. (Extended state space model)}

The states can be eliminated from the state space model equations (1) and (2) to yield the so called Extended State Space Model (ESSM)

$$
Y_{J+1 \mid L}=\tilde{A}_{L} Y_{J \mid L}+\tilde{B}_{L} U_{J \mid L+g}+\tilde{C}_{L} E_{J \mid L+1}
$$

where

$$
\begin{aligned}
& \tilde{A}_{L} \stackrel{\text { def }}{=} O_{L} A\left(O_{L}^{T} O_{L}\right)^{-1} O_{L}^{T} \quad \in \mathbb{R}^{L m \times L m} \\
& \tilde{B}_{L} \stackrel{\text { def }}{=}\left[O_{L} B \quad H_{L}^{d}\right]-\tilde{A}_{L}\left[\begin{array}{ll}
H_{L}^{d} & 0_{L m \times r}
\end{array}\right] \quad \in \mathbb{R}^{L m \times(L+g) r} \\
& \tilde{C}_{L} \stackrel{\text { def }}{=}\left[\begin{array}{ll}
O_{L} C & H_{L}^{s}
\end{array}\right]-\tilde{A}_{L}\left[\begin{array}{ll}
H_{L}^{s} & 0_{L m \times m}
\end{array}\right] \quad \in \mathbb{R}^{L m \times(L+1) m}
\end{aligned}
$$

Proof 3.10. Putting $J=: J+1$ in equation (37) and substituting $X_{J+1}=A X_{J}+$ $B U_{J}+C E_{J}$ into this equation gives

$$
Y_{J+1 \mid L}=O_{L} A X_{J}+\left[\begin{array}{ll}
O_{L} B & H_{L}^{d}
\end{array}\right] U_{J \mid L+g}+\left[\begin{array}{ll}
O_{L} C & H_{L}^{s}
\end{array}\right] E_{J \mid L+1}
$$

Equation (37) can be solved for $X_{J}$ when $(A, D)$ is observable, i.e.,

$$
X_{J}=O_{L}^{\dagger}\left(Y_{J \mid L}-H_{L}^{d} U_{J \mid L+g-1}-H_{L}^{s} E_{J \mid L}\right)
$$

where $O_{L}^{\dagger}=\left(O_{L}^{T} O_{L}\right)^{-1} O_{L}^{T}$ is the More-Penrose pseudo inverse of $O_{L}$. Substituting equation (93) into equation (92) gives equations (88)-(91).

Lemma 3.13. (Projection matrix for the deterministic subsystem)

Define the projection matrix

$$
Z_{J \mid L+1}^{d}=Y_{J \mid L+1} /\left[\begin{array}{l}
U_{J \mid L+g} \\
U_{0 \mid J} \\
Y_{0 \mid J}
\end{array}\right]
$$

This matrix can be partitioned into matrices $Z_{J+1 \mid L}^{d}$ and $Z_{J \mid L}^{d}$ which satisfy the deterministic model

$$
Z_{J+1 \mid L}^{a}=\tilde{A}_{L} Z_{J \mid L}^{d}+\tilde{B}_{L} U_{J \mid L+g}
$$

Proof 3.11. This follows from equations (88) and (13) and that

$$
\lim _{K \rightarrow \infty} \tilde{C}_{L} E_{J \mid L+1} /\left[\begin{array}{l}
U_{J \mid L+g} \\
U_{0 \mid J} \\
Y_{0 \mid J}
\end{array}\right]=0
$$

when the future inputs, $U_{J \mid L+g}$, the past data, $U_{0 \mid J}$ and $Y_{J \mid J}$, are all independent of the future noise term, $\tilde{C}_{L} E_{J \mid L+1}$. 
Lemma 3.14. (SID of the system matrices $B$ and $E$ )

Define from equation (95) the linear equation

$$
\mathscr{Y}=\tilde{B}_{L} \mathscr{U}
$$

where

$$
\begin{aligned}
& \mathscr{Y}=Z_{J+1 \mid L}^{d}-\tilde{A}_{L} Z_{J \mid L}^{d} \\
& \mathscr{U}=U_{J \mid L+g}
\end{aligned}
$$

From equation (97) a least squares problem

$$
\min _{B, E}\left\|\mathscr{Y}-\tilde{B}_{L}(B, E) \mathscr{U}\right\|_{F}^{2}
$$

for the unkown elements in $B$ and $E$ are defined as

$$
\operatorname{vec}(\mathscr{Y})=\mathscr{X} \operatorname{vec}\left(\left[\begin{array}{l}
B \\
E
\end{array}\right]\right)
$$

which can be solved for the unknown parameters as

$$
\operatorname{vec}\left(\left[\begin{array}{l}
B \\
E
\end{array}\right]\right)=\mathscr{X}^{\dagger} \operatorname{vec}(\mathscr{Y})
$$

where $\mathscr{X}^{\dagger}=\left(\mathscr{X}^{T} \mathscr{X}\right)^{-1} \mathscr{X}^{T}$. The matrix $\mathscr{X}$ is defined as follows

$$
\mathscr{X} \stackrel{\text { def }}{=} \sum_{i=1}^{L+g} R_{i}^{T} \otimes\left(E_{i-1}-\tilde{A}_{L} E_{i}\right) \in \mathbb{R}^{L m K \times(n+g m) r}
$$

where $\otimes$ denotes the Kronecker tensor product. The matrices $R_{i}$ and $E_{i}$ are defined in the following. The matrices

$$
R_{i} \in \mathbb{R}^{r \times K} \forall i=1, \ldots, L+g
$$

are $r$-block rows in the $\mathscr{U} \in \mathbb{R}^{(L+g) r \times K}$ matrix. I.e. extracted from

$$
\mathscr{U}=\left[\begin{array}{l}
R_{1} \\
\vdots \\
R_{L+g}
\end{array}\right]
$$

The matrices

$$
E_{i-1} \in \mathbb{R}^{L m \times(n+m g)} \forall i=1, \ldots, L+g
$$

are defined as follows:

$$
\begin{gathered}
E_{0}=\left[\begin{array}{ll}
O_{L} & 0_{L m \times m}
\end{array}\right], \quad E_{L+1}=0_{L m \times(n+m)} \\
E_{1}=\left[\begin{array}{ll}
0_{m \times n} & I_{m \times m} \\
D & 0_{m \times m} \\
D A & 0_{m \times m} \\
\vdots & \vdots \\
D A^{L-2} & 0_{m \times m}
\end{array}\right], E_{2}=\left[\begin{array}{ll}
0_{m \times n} & 0_{m \times m} \\
0_{m \times n} & I_{m \times m} \\
D & 0_{m \times m} \\
\vdots & \vdots \\
D A^{L-3} & 0_{m \times m}
\end{array}\right], E_{L}=\left[\begin{array}{ll}
0_{m \times n} & 0_{m \times m} \\
0_{m \times n} & 0_{m \times m} \\
0_{m \times n} & 0_{m \times m} \\
\vdots & \vdots \\
0_{m \times n} & I_{m \times m}
\end{array}\right]
\end{gathered}
$$

The matrix $I_{m \times m}$ denotes the $m \times m$ identity matrix. 
Proof 3.12. From equation (90) we have that $\tilde{B}_{L}$ is a linear function of $B$ and $E$ when $A$ and $D$ are given. The matrix $\mathscr{X}$ in the LS problem equation (101) is defined from $\tilde{B}_{L} \mathscr{U}$ by using the identity

$$
\operatorname{vec}(A X B)=\left(B^{T} \otimes A\right) \operatorname{vec}(X)
$$

Note that the number of columns in $\mathscr{Y}$ and $\mathscr{U}$, which is defined in equations (98) and (99), can be reduced to $K=:(L+g) r$ by post-multiplying both equations (98) and (99) with $U_{J \mid L+g}^{T}$. However, this does not affect the estimates of $B$ and $E$ but will in general reduce the computation. Another variant, which should be preferred for numerical reasons, is to define $y$ and $\mathscr{I}$ from the $R$ matrix provided by the $R Q / L Q$ decomposition. This will also reduce the number of columns to $K=:(L+g) r$ in Lemma 3.14.

Note that only a matrix of size $(n+g m) r \times(n+g m) r$ has to be inverted (i.e., the matrix $\mathscr{X}^{T} \mathscr{X}$ in the LS solution equation (102)) in order to solve for the unknown parameters in $B$ and $E$. This method combined with the LQ decomposition is found to be very efficient. The method in Lemma 3.14 require only that the input is exciting of order $n+g m$, and hence, independent of the user specified parameters $L$ and $J$. This is consistent with the lower bound on the order of persistence of excitation for consistent estimation of an $n$th order possibly proper $(g=1)$ linear system.

Note that the alternative strategy of first solving for $\tilde{B}_{L}$ in equation (95) and then extracting $B$ and $E$ would require the inversion of an $(L+g) r \times(L+g) r$ matrix $U_{J \mid L+g} U_{J \mid L+g}^{T}$. This matrix may be singular for colored input signals, and hence is not preferred.

\section{Closed loop subspace identification}

We have in Section 3.2 shown that the extended observability matrix $O_{L+1}$ can be estimated from the column space of the projection matrix $Z_{J \mid L+1}$ as defined in equation (52). Let us look at the error term in this projection. We have

$$
Z_{J \mid L+1}=O_{L+1} X_{J}^{a}+d Z
$$

The error term $d Z$ is given by

$$
\begin{aligned}
d Z & =H_{L+1}^{s}\left(E_{J \mid L+1} /\left[\begin{array}{l}
U_{J \mid L+g} \\
W
\end{array}\right]\right) U_{J \mid L+g}^{\perp} \\
& =H_{L+1}^{s} E_{J \mid L+1} U_{J \mid L+g}^{\perp} W^{T}\left(W U_{J \mid L+g}^{\perp} W^{T}\right)^{-1} W U_{J \mid L+g}^{\perp} \\
& \approx-H_{L+1}^{s} E_{J \mid L+1}^{\perp} / U_{J \mid L+g} W^{T}\left(W U_{J \mid L+g}^{\perp} W^{T}\right)^{-1} W U_{J \mid L+g}^{\perp}
\end{aligned}
$$

where $W$ is defined in equation (65). We have in the last expression in equation (111) used that $E_{J \mid L+1} W^{T} / K \approx 0$ when the number of columns $K$ tends to infinity. The remaining projection in the error term is then $E_{J \mid L+1} / U_{J \mid L+g}$. This term will also be approximately zero for open loop and many closed loop problems, which will be pointed out in the following. However, the term $E_{J \mid L+1} / U_{J_{\mid L+g}}$ may be non-zero and cause biased estimates for feedback systems in which the control is directly proportional to the innovations noise. We will in the next section discuss how to overcome this problem. We will also stress that biased estimates may be more reliable than estimates from an unbiased algorithm because the variance may be small. This is illustrated in the section of examples. 


\subsection{Closed loop subspace identification: Using a filter in the feeback loop!}

Since we are allowing the inputs to be colored the question whether it is possible with feedback in the inputs have to be pointed out. An external (dither) signal, i.e., a reference signal, should be used to excite the system when collecting data for subspace identification. The natural excitations from process disturbances are often insufficient. The SID algorithm works perfect for closed loop deterministic systems. Our simulation results also shows that the results may be good even for combined deterministic and stochastic systems, however, the results depends on the dither signal or the signal to noise ratio. If the signal to noise ratio is low then there may be a bias in the estimates. However, the variance may be small. There may also exist an 'optimal' dither signal which gives very accurate subspace estimates (small bias and small variance) even for systems with a large signal to noise ratio. This will be illustrated in Example 6.4. A white noise or random binary signal in the reference usually gives very good closed loop identification results. Furthermore, a minimum of measurements noise is, as always, to be preferred in order to obtain good closed loop estimates.

It is believed that SID of systems with state feedback or feedback from Kalman filter states would work well, provided an external dither signal is introduced in the loop. The reason for this is that the states are 'noise-free' and not correlated with the innovations noise. There are no problems by using subspace identification methods in these cases.

The key is to make the term $E_{J \mid L+1} / U_{J \mid L+g}$ small, which is equivalent to making the error term equation (39) small.

The (open loop) subspace identification methods may give biased estimates for closed loop systems as in Figures 1 and 3 when the signal-to-noise ratio is low. The reason for this is that the error term in equation (39) is not zero when the future inputs, $U_{J \mid L+g-1}$, are correlated with the future noise, $E_{J \mid L}$. Most of our simulations shows that the bias in the DSR algorithm, due to noisy closed loop data, is less than the bias in the other algorithms as N4SID, SUBID (Van Overschee and De Moor (1996)), MOESP (Verhagen (1994)).

One of our solutions to the bias problem is to include a filter, e.g. a first order low-pass filter, in the feedback path of the control system as illustrated in Figure 2. This filter will eliminate the feedback problem when using subspace identification

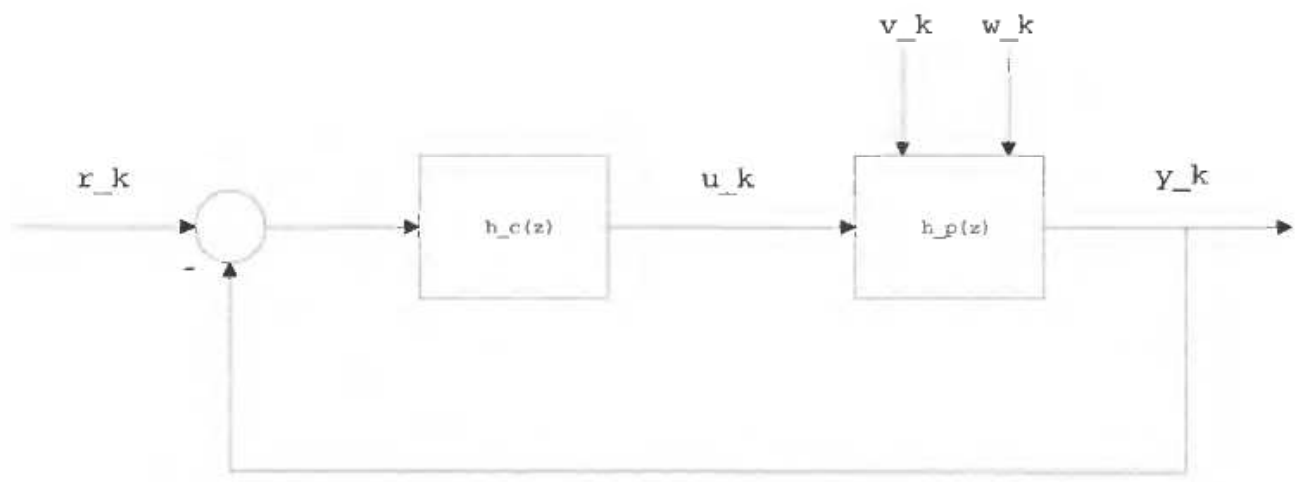

Figure 1. Standard feedback system with reference signal, $r_{k}$, controller represented with $u_{k}=h_{c}(z)\left(r_{k}-y_{k}\right)$ and the system represented with $h_{p}(z)$. Possibly process and measurements noise are represented with $v_{k}$ and $w_{k}$, respectively. 


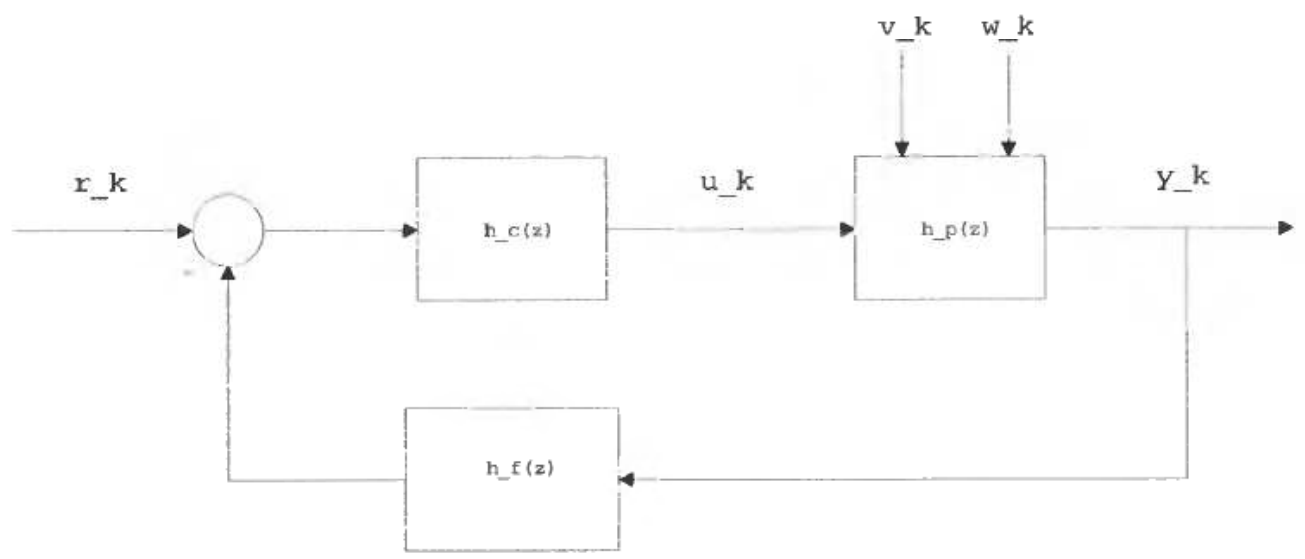

Figure 2. Feedback system with a filter in the feeback loop in order to eliminate problems with feeback in the data when using subspace identification algorithms. Here, $u_{k}$ is the control/ input signal, $y_{k}$ the output signal and $r_{k}$, is the reference signal. The controller is represented with $u_{k}=h_{c}(z)\left(r_{k}-\bar{y}_{k}\right)$ where $\bar{y}_{k}=h_{f}(z) y_{k}$ is the filtered output. The system is represented with the transfer function $h_{p}(z)$. The controller is represented with the transfer function $h_{c}(z)$ and the filter is represented with the transfer function $h_{f}(z)$. Possibly process and measurements noise are represented with $v_{k}$ and $w_{k}$, respectively.

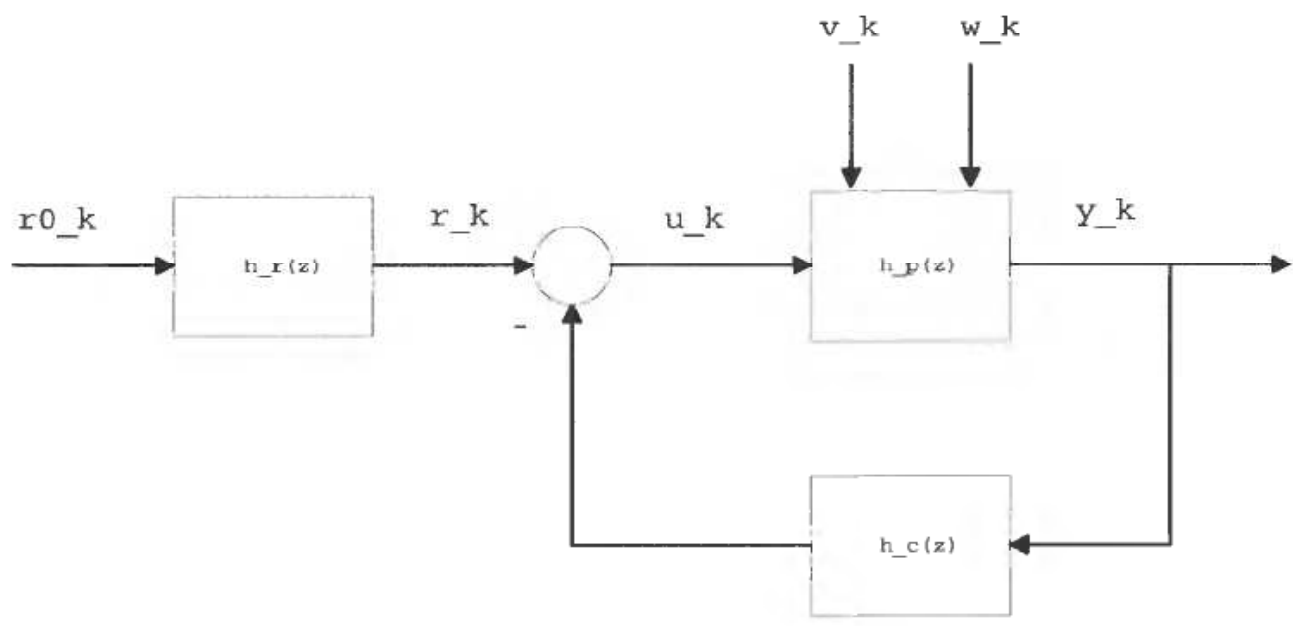

Figure 3. Alternative feedback system with reference signal, $r_{k}$, controller represented with $u_{k}=r_{k}-h_{c}(z) y_{k}$ and the system represented with $h_{p}(z)$. Possibly process and measurements noise are represented with $v_{k}$ and $w_{k}$, respectively.

algorithms. Hence, the input and output data, $u_{k}$ and $y_{k}$, can be used directly to identify a state space model of the system. The reason for this is that the input is no longer directly proportional to the measurements noise $w_{k}$ in the output $y_{k}=D x_{k}+w_{k}$ (or the innovations $e_{k}$ in the output $y_{k}=D x_{k}+e_{k}$ ). This solution to the feedback problem will be illustrated in Example 6.3.

Note that the control system in Figure 2 is a special case of the more general control configuration in Figure 3.

Note that SID algorithms are very useful for model predictive control in which 
the control input signal often is the reference signal to some local controller for the process. Hence, the SID algorithm are used to identify the closed loop model from the reference signal to the output. In this case we do not have problems with feedback in the data.

\subsection{Closed loop subspace identification: Using the controller}

The knowledge of the controller or the reference signal can be used to obtain consistent subspace identification algorithms for closed loop systems. Consider a linear state space model of the controller in Figure 1, i.e.

$$
\begin{gathered}
x_{k+1}^{c}=A_{c} x_{k}^{c}+B_{c}\left(r_{k}-y_{k}\right) \\
u_{k}=D_{c} x_{k}^{c}+E_{c}\left(r_{k}-y_{k}\right)
\end{gathered}
$$

where $A_{c} \in \mathbb{R}^{n_{c} \times n_{c}}, B_{c} \in \mathbb{R}^{n_{c} \times m}, D_{c} \in \mathbb{R}^{r \times n_{c}}$ and $E_{c} \in \mathbb{R}^{r \times m}$ is the state space model matrices of the controller and $x_{k}^{c} \in \mathbb{R}^{n}$ is the controller state vector. Note also that the results which follows also holds for the control strategy in Figure 3.

We will in the following assume that the controller is linear and that the quadruple $\left(A_{c}, B_{c}, D_{c}, E_{c}\right)$ matrices in addition to the input and output data, $u_{k}$ and $y_{k}$, are known. One should also note that the linear controller matrices can be exactly identified if $r_{k}, u_{k}$ and $y_{k}$ are known. The problem of identifying the controller is deterministic and one can in this case perfectly identify $\left(A_{c}, B_{c}, D_{c}, E_{c}\right)$ by using $r_{k}-y_{k}$ as inputs and $u_{k}$ as outputs by using e.g. the DSR subspace algorithm (provided that noise-free $r_{k}$ 's and $u_{k}$ 's are given).

Consider the following matrix equation obtained from the state space model equations (112) and (113) of the controller in Figure 1, i.e.

$$
U_{J \mid L}=O_{L}^{c} X_{J}^{c}+H_{L}^{c}\left(R_{J \mid L}-Y_{J \mid L}\right)
$$

We will now adopt the idea in Van Overschee and De Moor (1997) and define a signal/matrix $M_{J \mid L}$ from equation (114) which is orthogonal to the future noise matrix $E_{J \mid L}$ as

$$
M_{J \mid L} \stackrel{\text { def }}{=} U_{J \mid L}+H_{L}^{c} Y_{J \mid L}=O_{L} X_{J}^{c}+H_{L}^{c} R_{J \mid L}
$$

The signal/matrix equation (115) was introduced by Van Overschee and De Moor (1997) in order to solve the bias problem in the subspace identification algorithms.

Note also that a similar signal can be defined from the control system in Figure 3. The only difference is that the right hand side of equation (115) becomes $R_{J \mid L}-O_{L} X_{J}^{c}$ in this case. This matrix is also orthogonal to the future noise and the closed loop subspace identification algorithm which will be presented in the following thus holds for both control systems as presented in Figures 1 and 3. The main point of introducing $M_{J \mid L}$ is that

$$
E_{J \mid L} / M_{J \mid L}=0
$$

which holds if $E_{J \mid L}$ is orthogonal to both $X_{J}^{c}$ and $R_{J \mid L}$.

\subsection{Closed loop subspace identification: Indirect method}

We will in this section derive a consistent version of the closed loop subspace identification algorithm which is presented in Van Overschee and De Moor (1997). We have the following consistent projection lemma for closed loop subspace system identification. 
Lemma 4.1. (Closed loop SID)

Given the following closed loop projection

$$
Z_{J \mid L+1}=\left(Y_{J \mid L+1} /\left[\begin{array}{l}
M_{J \mid L+g} \\
U_{0 \mid J} \\
Y_{0 \mid J}
\end{array}\right]\right) M_{J \mid L+g}^{\perp}
$$

Then, $Z_{J \mid L+1}$ is related to the extended observability matrix $O_{L+1}$ as

$$
Z_{J \mid L+1}=T^{-1} O_{L+1} X_{J}^{a}
$$

where $T \in \mathbb{R}^{(L+1) m \times(L+1) m}$ is a lower block Toepliz matrix given by

$$
\begin{array}{ll}
T=I_{(L+1) m}+H_{L+1}^{d} H_{L+g}^{c} & \text { when } g=1 \\
T=I_{(L+1) m}+\left[\begin{array}{ll}
H_{L+1}^{d} H_{L+g}^{c} & 0_{(L+1) m \times m}
\end{array}\right] & \text { when } g=0
\end{array}
$$

Furthermore, the system order is given by

$$
n=\operatorname{rank}\left(Z_{J \mid L+1}\right)
$$

From the SVD

$$
\mathrm{Z}_{J \mid L+1}=U_{1} S_{1} V_{1}^{T}+U_{2} S_{2} V_{2}^{T}
$$

where the $n$ large/dominant singular values are contained on the diagonal in $S_{1}$ and the other zero/smaller singular values on the diagonal of $S_{2}$. Furthermore, $U_{1} \in \mathbb{R}^{(L+1) m \times n}$ and $U_{2} \in \mathbb{R}^{(L+1) m \times((L+1) m-n)}$.

From this we have the estimate

$$
T^{-1} O_{L+1}=U_{1}
$$

Furthermore, the 'autonomous' states are determined as $X_{J}^{a}=S_{1} V_{1}^{T}$.

Proof 4.1. A simple proof is given as follows. From equation (26) we have

$$
Y_{J \mid L+1}=O_{L+1} X_{J}+H_{L+1}^{d} U_{J \mid L+g}+H_{L+1}^{s} E_{J \mid L+1}
$$

Adding $H_{L+1}^{d} H_{L+g}^{c} Y_{J \mid L+g}$ on both sides of equation (124) and using the definition in equation (115) gives

$$
T Y_{J|L| 1}=O_{L, 1} X_{J}+H_{L, 1}^{d} M_{J \mid L+g}+H_{L+1}^{s} E_{J \mid L+1}
$$

where $T$ is as in equations (119) and (120). Since the matrix

$$
\left[\begin{array}{l}
M_{J \mid L+g} \\
U_{0 \mid J} \\
Y_{0 \mid J}
\end{array}\right]
$$

is uncorrelated (orthogonal) to the future noise matrix $E_{J \mid L+1}$ we have that

$$
T Z_{J \mid L+1}^{d}=O_{L+1} X_{J}^{d}+H_{L+1}^{d} M_{J \mid L+g}
$$

where

$$
\mathrm{Z}_{J \mid L+1}^{d}=: Y_{J \mid L+1} /\left[\begin{array}{l}
M_{J \mid L+g} \\
U_{0 \mid J} \\
Y_{0 \mid J}
\end{array}\right]
$$

and 


$$
X_{J \mid L+1}^{d}=: X_{J} /\left[\begin{array}{l}
M_{J \mid L+g} \\
U_{0 \mid J} \\
Y_{0 \mid J}
\end{array}\right]
$$

The lower triangular matrix $T$ is non-singular if $I_{m}+E E_{c}$ is non-singular. Hence,

$$
Z_{J \mid L+1}^{d}=T^{-1} O_{L+1} X_{J}^{d}+T^{-1} H_{L+1}^{d} M_{J \mid L+g}
$$

Post-multiplication of equation (129) with $M_{J \mid L+g}^{\perp}$ proves equations (117)-(119).

Furthermore we have that

$$
U_{2}^{T} Z_{J \mid L+1}^{d}=U_{2}^{T} T^{-1} H_{L+1}^{d} M_{J \mid L+g}
$$

where $U_{2}$ is the left singular vectors from the SVD of $Z_{J \mid L+1}$ which is related to the 'zero/small' singular values. Equation (130) is obtained by pre-multiplying equation (129) with $U_{2}^{T}$ and using that $T^{-1} O_{L+1}=U_{1}$ and $U_{2}^{T} U_{1}=0$.

Equation (130) is a linear equation of the elements in the lower block triangular Toepliz matrix $T^{-1} H_{L+1}^{d}$. The solution to this problem is an important step in the closed loop subspace algorithm and therefore needs further discussion. In order to do this we write equation (130) as

$$
\mathscr{Y}=\mathscr{U} \mathscr{K} M_{J \mid L+g}
$$

where

$$
\begin{gathered}
\mathscr{Y}=: U_{2}^{T} Z_{J \mid L+1}^{d} \\
\mathscr{U}=: U_{2}^{T} \\
\mathscr{K} \stackrel{\text { def }}{=} T^{-1} H_{L+1}^{d} \in \mathbb{P}^{(L+1) m \times(L+g) r}
\end{gathered}
$$

The matrix $\mathscr{K}$ is a lower block triangular Toepliz matrix with $m \times r$ blocks $K_{i}$ $\forall i=1, \ldots, L+1$. Hence, we can solve equation (130) (or equivalent equation (131) in a least squares optimal sense for the parameters in $\mathscr{K}$. An algorithm for doing this is presented in Van Overschee and De Moor (1996b) Appendix C.

The problem of solving equation (130) is very similar to the least squares problem in Section 3.4 for determining $B$ and $E$ for the open loop subspace problem.

Since $H_{L+g}^{c}$ is known the matrix $H_{L+1}^{d}$ is simply obtained from $\mathscr{K}$ as (when $g=1)$

$$
H_{L+1}^{d}=\mathscr{K}\left(I_{(L+1) r}-H_{L+g}^{c} \mathscr{K}\right)^{-1}
$$

Finally, the extended observability matrix of the system can be obtained as (when $g-1)$

$$
O_{L+1}=\left(I_{(L+1) m}+H_{L+1}^{\mathrm{d}} H_{L+g}^{c}\right) U_{1}
$$

The system matrices $A$ and $D$ are then computed from $O_{L+1}$. The system matrices $B$ and $E$ are computed from $H_{L+1}^{d}$ and $O_{L}$.

The stochastic part of the system is determined very similar to the theory in Section 3.3. The difference is that the projection matrix now is given by

$$
Z_{J \mid L+1}^{s}=Y_{J \mid L+1}-Y_{J \mid L+1} /\left[\begin{array}{l}
M_{J \mid L+g} \\
U_{0 \mid J} \\
Y_{0 \mid J}
\end{array}\right]=T^{-1} H_{L+1}^{s} E_{J \mid L+1}
$$


The projection matrices $Z_{J \mid L+1}, Z_{J \mid L+1}^{d}$ and $Z_{J \mid L+1}^{s}$ can effectively be computed by the LQ/QR decomposition.

The above ideas is among other details used to construct a MATLAB function, $d s r_{-} c l . m$, for consistent closed loop subspace identification.

\subsection{Closed loop subspace identification: Direct method}

A drawback with the above algorithm is that $T$ and $H_{L+1}^{d}$ have to be identified before the extended observability matrix $O_{L+1}$ and the system matrices $A$ and $D$ could be identified. We will in this section present a solution to the closed loop subspace identification problem which is more consistent with the DSR subspace algorithm in which $O_{L+1}$ and $A$ and $D$ is identified directly in a first step. We have the following lemma.

\section{Lemma 4.2. (Direct closed loop SID)}

The extended observability matrix $O_{L+1}$ is obtained from the following projection

$$
\begin{aligned}
Z_{J \mid L+1} & =Z_{J|L| 1}^{d}\left(M_{J \mid L+g}-H_{L+1}^{c} Z_{J \mid L+1}^{d}\right)^{\perp} \\
& =Z_{J \mid L+1}^{d}\left(U_{J \mid L+g}+H_{L+1}^{c} Z_{J \mid L+1}^{s}\right)^{\perp}=O_{L+1} X_{J}^{a}
\end{aligned}
$$

where $Z_{J \mid L+1}^{d}$ and $Z_{J \mid L+1}^{s}$ are defined in equations (127) and (137), respectively.

Furthermore, $B$ and $E$ (or also $H_{L+1}^{d}$ ) can be obtained from

$$
Z_{J \mid L+1}^{d}=O_{L+1} X_{J}^{d}+H_{L+1}^{d}\left(M_{J \mid L+g}-H_{L+1}^{c} Z_{J \mid L+1}^{d}\right)
$$

or from (as in the standard DSR algorithm) the equation

$$
Z_{J+1 \mid L+1}^{d}-\tilde{A}_{L} Z_{J \mid L}=\tilde{B}_{L}\left(M_{J \mid L+g}-H_{L+1}^{c} Z_{J \mid L+1}^{d}\right)
$$

Proof 4.2. From equation (126) we have that

$$
\left(I+H_{L+1}^{d} H_{L+g}^{c}\right) Z_{J \mid L+1}^{d}=O_{L+1} X_{J}^{d}+H_{L+1}^{d} M_{J \mid L+g}
$$

Rearranging equation (141) gives

$$
Z_{J \mid L+1}^{d}=O_{L+1} X_{J}^{d}+H_{L+1}^{d}\left(M_{J \mid L+g}-H_{L+g}^{c} Z_{J \mid L+1}^{d}\right)
$$

The closed loop subspace algorithm which is presented in this section is very similar to the open loop subspace identification algorithm which is presented in Section 3 . The only difference is that the projection matrices are modified to incorporate the Markov parameters of the controller (the Toepliz matrix $H_{L+g}^{c}$.

\section{Further remarks}

\subsection{Choice of algorithm parameters}

There are two parameters in the algorithm, i.e., $L$ and $J . L$ is interpreted as the identification-horizon used to predict the number of states. $J$ is interpreted as the horizon (into the past) used to define instruments from the data which are used to remove noise. The system order, $n$, which is specified or identified, is bounded by the user-specified parameter $L$, i.e. so that, $1 \leqslant n \leqslant m L$ where $m$ is the number of outputs. Hence, $L m$ singular values are computed by the algorithm and the user may chose the system order by inspection of the non-zero singular values. 
A rule of thumb is that $L$ should be chosen as small as possible if the inputs are poorly exciting. The minimum identification-horizon, $L_{\min }$, so that the pair $(D, A)$ is observable and $\operatorname{rank}\left(O_{L_{\min }}\right)=n$ is bounded by, $\lceil n / m\rceil \leqslant L_{\min } \leqslant n-\operatorname{rank}(D)+1$, where $\lceil\cdot\rceil$ is the ceiling function, i.e., rounding towards plus infinity. If the outputs are independent, then, we suggest to use $L_{\min }=n-m+1$ when $n \geqslant m$ and $L_{\min }=1$ when $n=m$. If the inputs are rich, e.g. white, then this point is not critical. In practice, it is suggested that model validation on independent data is taken into consideration when choosing the 'optimal' settings for $L$.

The past horizon, $J$, may for combined deterministic and stochastic systems and for pure stochastic systems usually be chosen as $J=L+1$ or $J=L$. Note that the estimates of $C$ and the Kalman filter gain matrix $K=C F^{-1}$ usually becomes better when $J$ increases. For pure deterministic systems we may chose $J=1$. The instruments $Y_{0 \mid J}$ and $U_{0 \mid J}$ can also be removed from the projections, i.e., and putting $J=0$, in this case.

\subsection{Choice of input signal}

The subspace identification methods tends to be more sensitive to the input signal compared to e.g, the Prediction Error Method (PEM). This means that there may exist colored input signals which gives subspace estimates which are as optimal (efficient and consistent) as the PEM estimates. On the other side there may exist colored input signals where the subspace methods gives poorer results compared to the PEM. An optimal experiment for the subspace methods is in general not a white noise input, but rather a colored input signal where the frequency spectrum is optimized to excite the parameters in the system as well as possible. Our experience is also that an input signal which are minimizing the condition number of the Hankal matrix $U_{0 \mid J}$ or $U_{J \mid L+g}$, is usually not an optimal input signal.

\subsection{N4SID}

The N4SID algorithms in Van Overschee and De Moor (1994) are using an oblique-projection

$$
\begin{aligned}
\mathcal{O}_{i} & =Y_{i \mid i} U_{i \mid i}^{\perp} W_{p}^{T}\left(W_{p} U_{i \mid i}^{\perp} W_{p}^{T}\right)^{-1} W_{p} \\
W_{p} & =\left[\begin{array}{c}
U_{0 \mid i} \\
Y_{0 \mid i}
\end{array}\right]
\end{aligned}
$$

for the identification of the extended controllability matrix $O_{i}$, i.e., $O_{i}$ is estimated from the column space of $\mathcal{O}_{i}$ in equation (143), e.g. using the SVD. Comparing equation (64) with equation (143) shows that the extra projection matrix $U_{i \mid i}^{\perp}$ are missing on the left hand side of equation (143). Hence, we conclude that in general

$$
\mathcal{O}_{i} \neq O_{i} X_{i}^{a}
$$

The consequence of this is that the subspace identification theorems in Van Overschee and De Moor (1994), (1996), (1997) which are using the oblique projection, to our understanding, are wrong.

The extra projection matrix $U_{i \mid i}^{\perp}$ on the left hand side of equation (143) removes the influence of the future inputs on the future outputs, and is necessary in order to obtain accurate/consistent subspace estimates for colored input signals. Hence, a consistent projection is therefore

$$
Z_{i \mid i}=\mathcal{O}_{i} U_{i \mid i}^{\perp}=O_{i} X_{i}^{a} U_{i \mid i}^{\perp}
$$


Hence, the extra projection can not be considered as a weighting matrix but as a projection matrix. One should note that the parameter $i$ used by N4SID is related to the parameter $L$ in DSR as $i=L+1$. Furthermore, in N4SID the past horizon is put equal to the future horizon. Hence, this corresponds to putting $J=L+1=i$ in DSR.

\section{Numerical examples}

\subsection{Example 1}

Given the system (1) and (2) with the following matrices and vectors

$$
\begin{gathered}
A=\left[\begin{array}{cc}
0 & 1 \\
-0.7 & 1.5
\end{array}\right], \quad B=\left[\begin{array}{l}
0.25 \\
0.625
\end{array}\right], \quad C=\left[\begin{array}{l}
0.5 \\
0.5
\end{array}\right], \\
D=\left[\begin{array}{ll}
1 & 0
\end{array}\right], \quad E=1, F=1
\end{gathered}
$$

The following colored input signals where used for identification

$$
\begin{aligned}
& u_{k}^{1}=\sin (k)+\sin \left(\frac{k}{2}\right), \\
& u_{k}^{2}=\sin (k)+\sin \left(\frac{k}{2}\right)+\sin \left(\frac{k}{3}\right) \\
& u_{k}^{3}=\text { with noise with variance } E\left(u_{k}^{2}\right)=1
\end{aligned}
$$

The number of samples was $N=1000$. The system was simulated 100 times, each time with the same input but with a different noise realization $e_{k}$. However, with the same covariance $E\left(e_{k}^{2}\right)=1$. The DSR parameters where chosen as $L=2, J=3$. The model structure parameter where $g=1$. The poles of the 100 identified system matrices are illustrated in Figures 4, 5 and 6. From this we conclude that the method presented in this paper is almost as efficient as the PEM method, for the inputs which are considered. However, the N4SID obliquc-method gives an unacceptable bias in the pole estimates for input $u_{k}^{1}$, i.e., because the estimated poles are unstable. From Figure 5 we see that the bias is eliminated but the results from N4SID are highly uncertain. However, the results from N4SID are nearly the same as DSR for the white input signal $u_{k}^{3}$. We also see that the variance of the subspace estimates may be smaller for a colored input signal, Figure 5, than for the white noise input, Figure 6.

\subsection{Example 2}

We consider the following system

$$
\begin{aligned}
x_{k+1} & =A x_{k}+B u_{k}+C v_{k} \\
y_{k} & =D x_{k}+w_{k}
\end{aligned}
$$

where the system matrices are the same as in Example 6.1. The process noise, $v_{k}$, and the measurements noise, $w_{k}$, are both white noise with standard deviation $\sqrt{E\left(v_{k}^{2}\right)}=\sqrt{0.02}=0.1458$ and $\sqrt{E\left(w_{k}^{2}\right)}=\sqrt{0.002}=0.0447$, respectively. The system is 

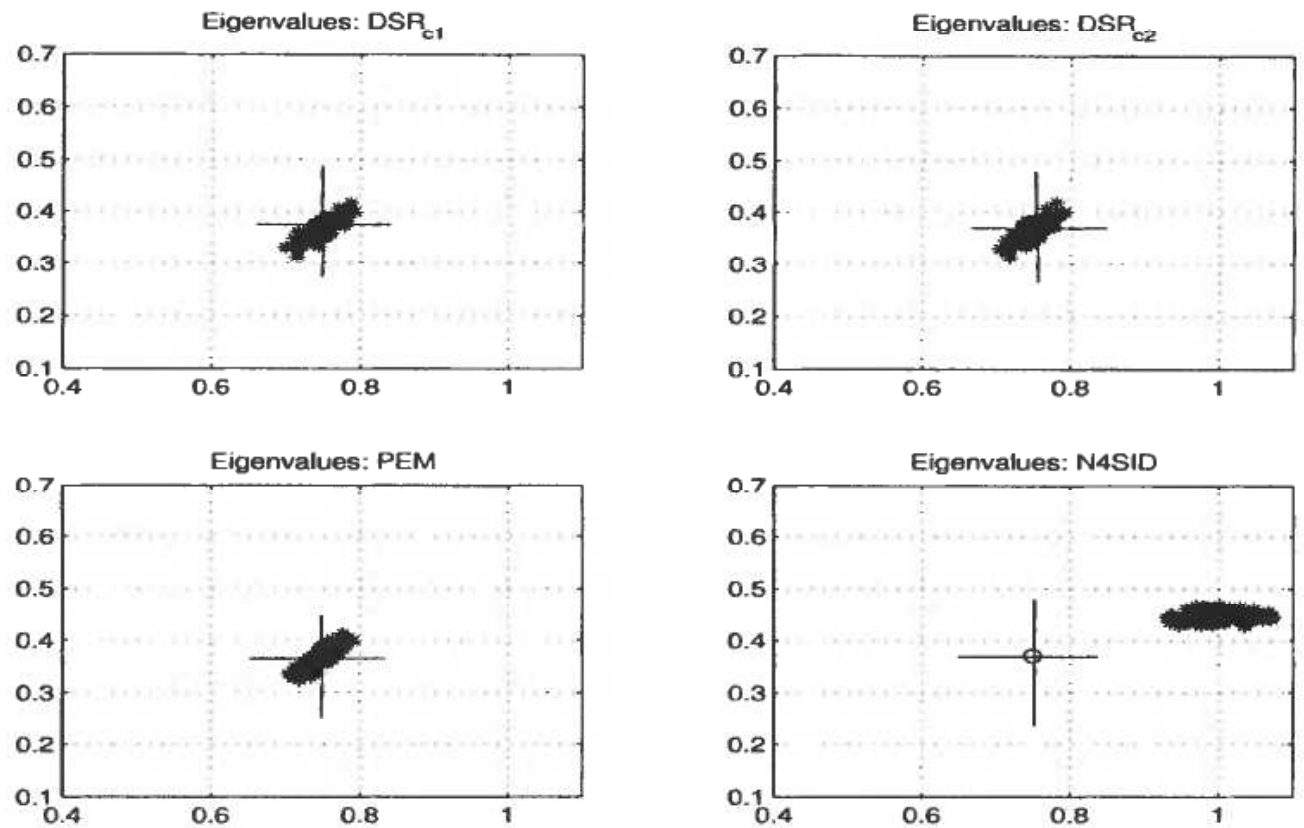

Figure 4. Identified poles for a Monte carlo simulation. The exact pole is marked with a cross. Input signal $u_{k}^{1}$ where used.
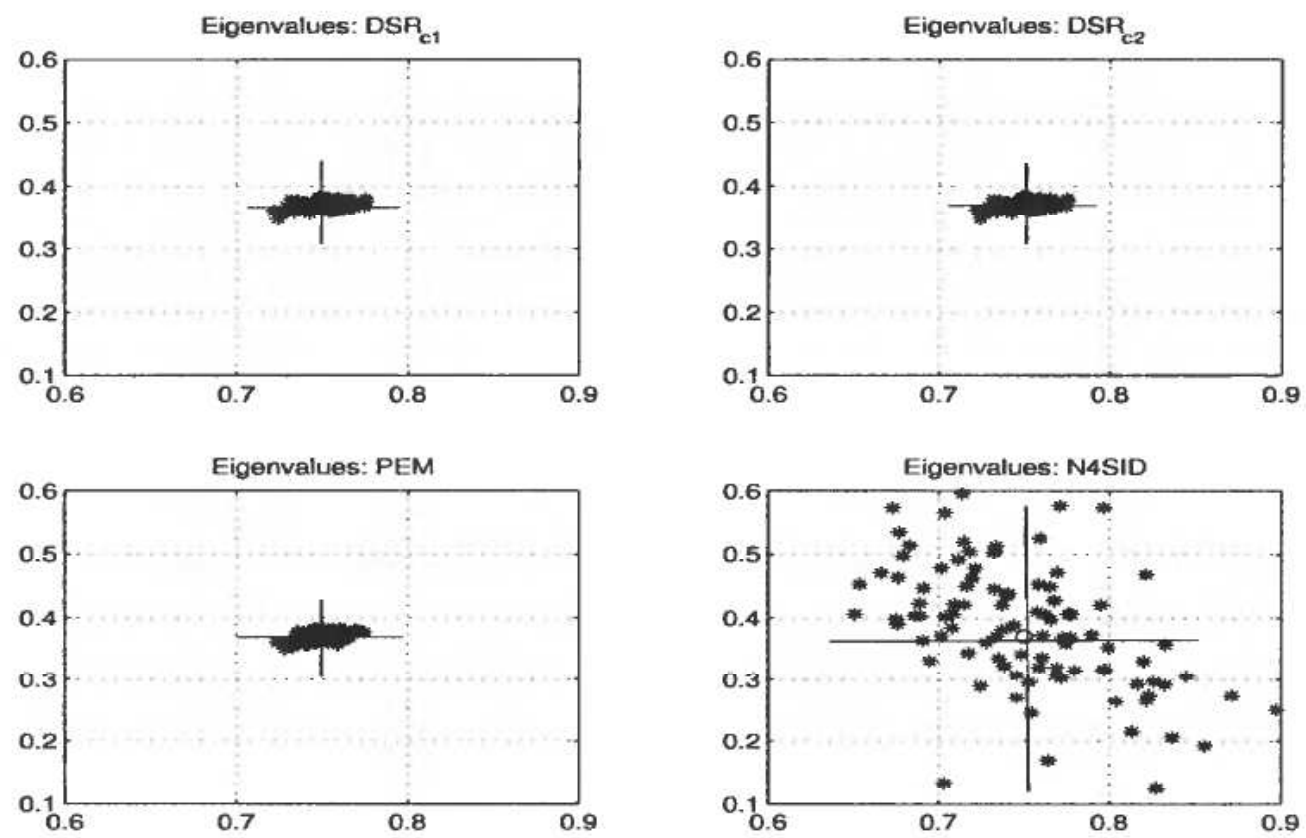

Figure 5. Identified poles for a Monte carlo simulation. The exact pole is marked with a cross. Input signal $u_{k}^{2}$ where used. 

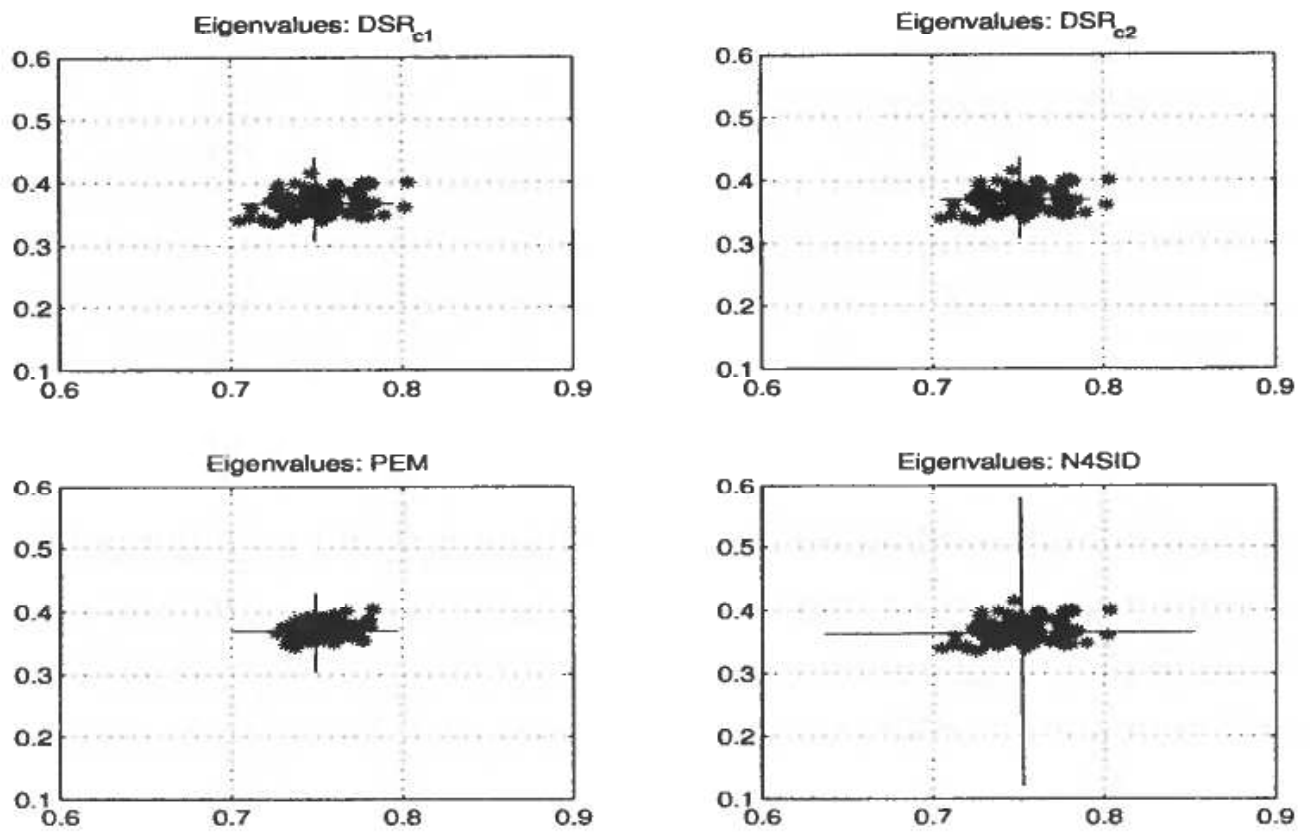

Figure 6. Identified poles for a Monte carlo simulation. The exact pole is marked with a cross.Input signal $u_{k}^{3}$ where used in this case.

operating in closed loop. The input to the system is generated by the following discrete time PI-controller

$$
u_{k}=K_{p}\left(r_{k}-y_{k}\right)+z_{k}
$$

where the controller state, $z_{k}$, is defined by

$$
z_{k+1}=z_{k}+\frac{K_{p}}{T_{i}}\left(r_{k}-y_{k}\right)
$$

The proportional constant is $K_{p}=0.2$, the integral time is $T_{i}=5$ and the reference, $r_{k}$, is taken as the binary signal in Figure 7.

The number of samples was $N=1000$. The system was simulated 100 times, each time with the same reference, $r_{k}$, but with a different noise realizations $v_{k}$ and $w_{k}$, but with the same variance. The DSR parameters where chosen as $L=J=3$ and the structure parameter where $g=0$. The subspace algorithms works perfect in the deterministic case. However, the algorithm gives a small bias in the estimates in the case of noise. The bias is negligible for this example. The pole estimates are presented in Figure 8.

\subsection{Example 3}

Consider the same closed loop example as in Example 2. We will in this example illustrate the bias-problem when using subspace identification algorithms directly from input and output data collected in closed loop (Figure 1). Furthermore, we will illustrate that the feedback problem can be eliminated by including a low-pass filter in the feedback as in Figure 2.

The process noise, $v_{k}$, and the measurements noise, $w_{k}$, are both white noise with 

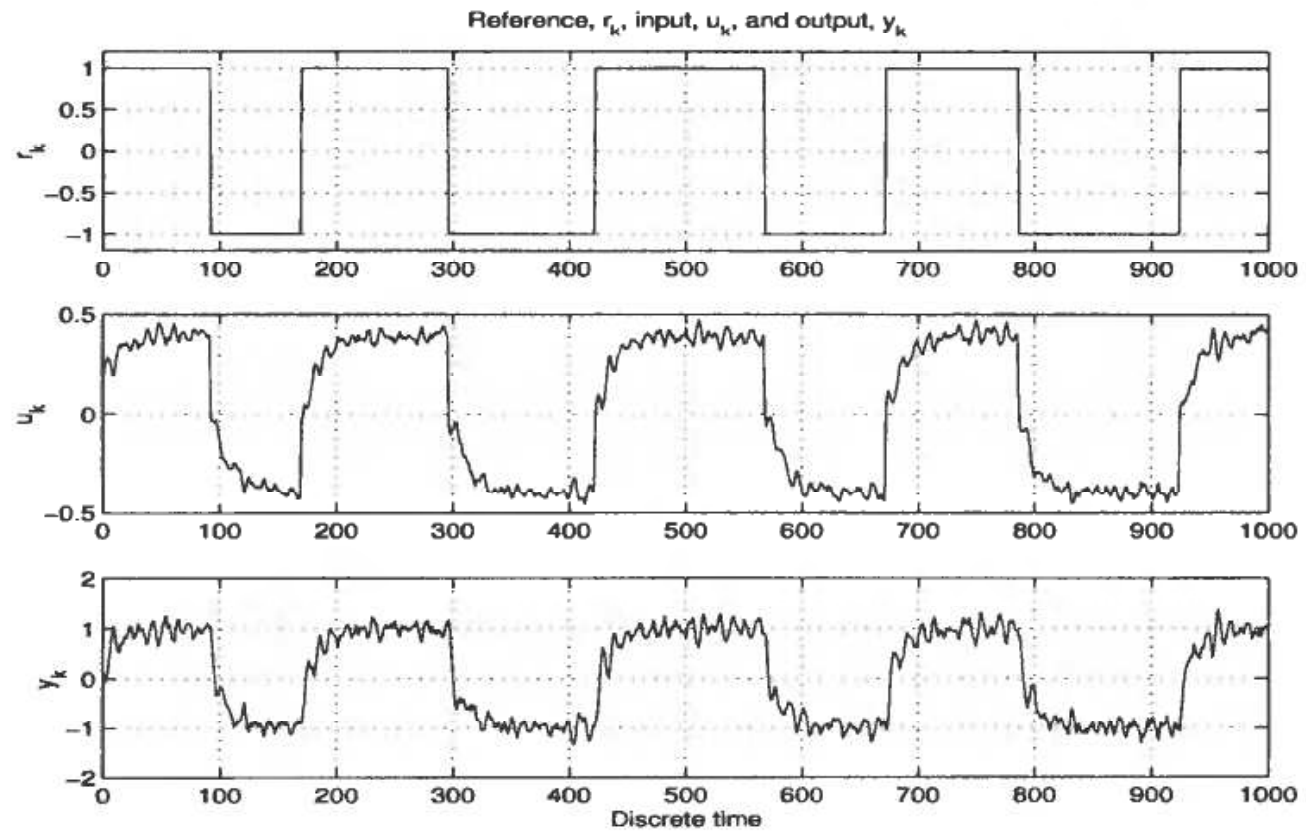

Figure 7. The reference signal, $r_{k}$, the input, $u_{k}$ and the output $y_{k}$ for two particular noise realizations $v_{k}$ and $w_{k}$, used in Example 6.2.
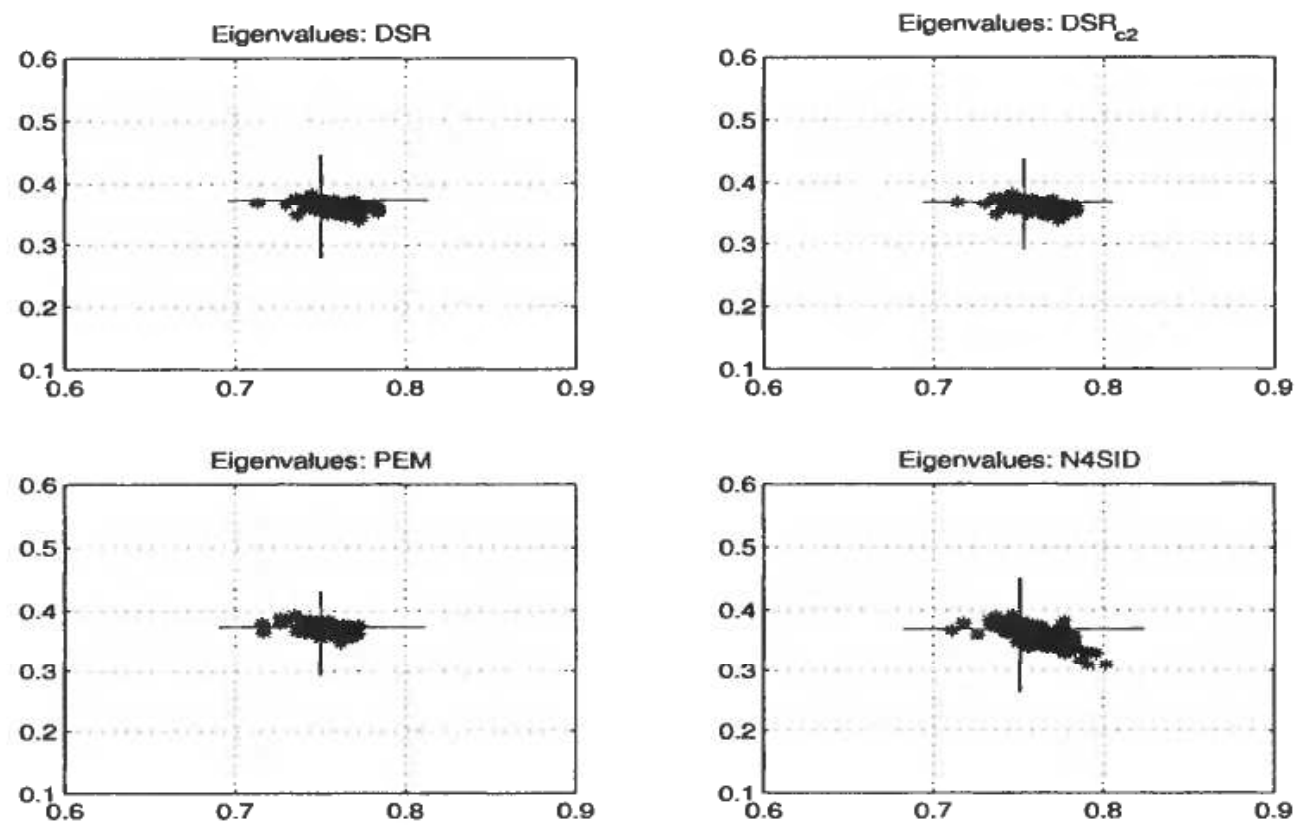

Figure 8. The pole estimates from the closed loop data as described in Example 6.2. 

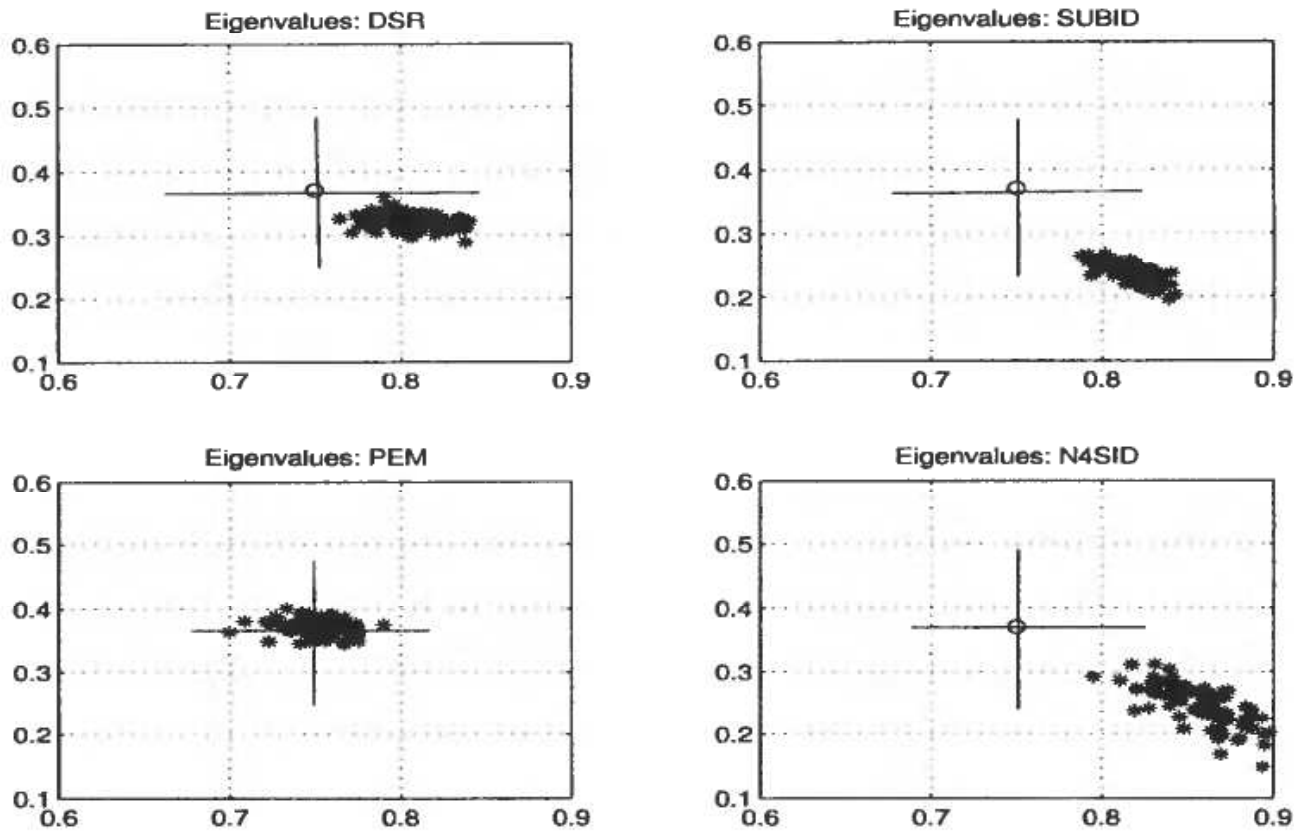

Figure 9. The pole estimates from the closed loop data as described in Example 6.3. The control system is as in Figure 1 with the same reference signal as in Figure 7.

standard deviation $\sqrt{E\left(v_{k}^{2}\right)}=\sqrt{0.05}=0.2236$ and $\sqrt{E\left(w_{k}^{2}\right)}=\sqrt{0.01}=0.1$, respectively. The pole estimates after a Monte carlo simulation is presented in Figure 9. We can clearly see a bias in the estimates from the (open loop) subspace identification algorithms. The bias in the DSR estimates is smaller than the bias in the estimates from both SUBID (Van Overschee and De Moor (1996)) and N4SID. This is also the conclusion from many other simulation experiments.

Consider now the feedback system in Figure 2. We are using a PI-controller as in Example 6.2 where the filtered output is used as input to the controller. The controller equations are as follows.

$$
u_{k}=K_{p}\left(r_{k}-\bar{y}_{k}\right)+z_{k}
$$

where the controller state, $z_{k}$, is defined by

$$
z_{k+1}=z_{k}+\frac{K_{p}}{T_{i}}\left(r_{k}-\bar{y}_{k}\right)
$$

The filter is a 1. order low-pass filter of the form

$$
\begin{aligned}
\bar{y}_{k+1} & =\bar{y}_{k}+K_{f}\left(y_{k}-\bar{y}_{k}\right) \\
& =\left(1-K_{f}\right) \bar{y}_{k}+K_{f} y_{k}
\end{aligned}
$$

with filter constant $K_{f}=0.1$. The initial filter output is taken as $\bar{y}_{0}=y_{0}$. Pole estimates after a Monte Carlo simulation is illustrated in Figure 10. We see that the pole estimates now are consistent.

\subsection{Example 4}

We will in this example search for an optimal experiment in the reference. Consider the reference $r_{k}=\sin (\omega k)$ for varying frequency $\omega$. The following investigation shows 

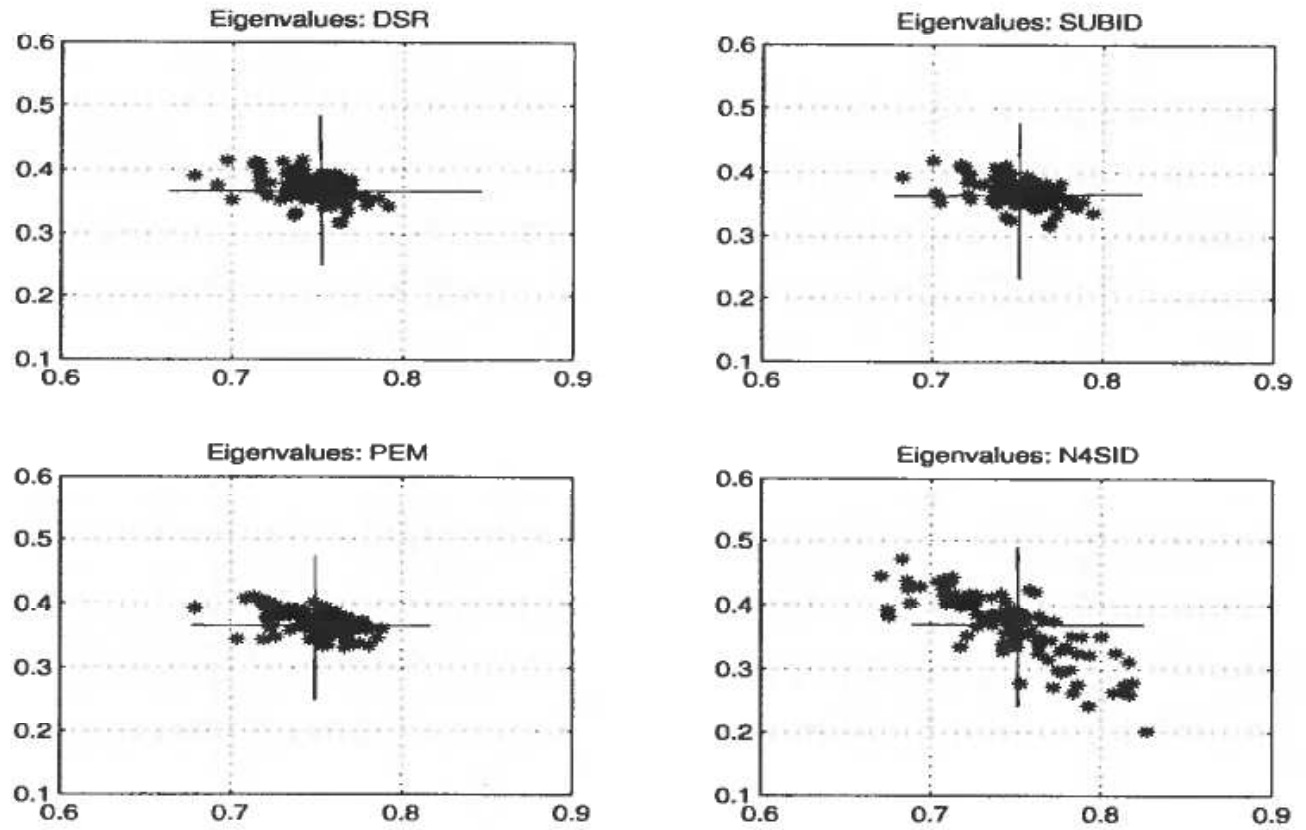

Figure 10. The pole estimates from the closed loop data as described in Example 6.3 with a filter in the feedback. The control system is as in Figure 2.

that the bias in the DSR pole estimates is a function of the frequency and that the bias reach a minimum for a particular frequency.

The reference signal which gave the smallest bias in the pole estimates is for this example found to be

$$
r_{k}=\sin \left(\frac{1}{1.526} \omega\right)
$$

The pole estimates from an Monte carlo experiment is illustrated in Figure 11. The results are very interesting because, as we see, the pole estimates from the DSR subspace identification method is more reliable than the pole estimates from the prediction error method PEM.

The process noise, $v_{k}$, and the measurements noise, $w_{k}$, are both white noise with standard deviation $\sqrt{E\left(v_{k}^{2}\right)}=\sqrt{0.1}=0.01$ and $\sqrt{E\left(w_{k}^{2}\right)}=\sqrt{0.1}=0.01_{k}$, respectively. The DSR parameters is $L=5, g=0$ and $J=6$.

\section{Conclusion}

The extended observability matrix $O_{L+1}$ can be computed directly from the column space of a projection matrix $Z_{J \mid L+1}$, which is defined in terms of the known data. There are in general two projections involved in order to define $Z_{J_{\mid L+1}}$. One projection is used to remove the effect of noise and one projection is used to remove the effect of future inputs from the future outputs. A necessary condition for a consistent estimate of $O_{L+1}$ is that the number of columns $K$ in the data matrices tends to infinity. 

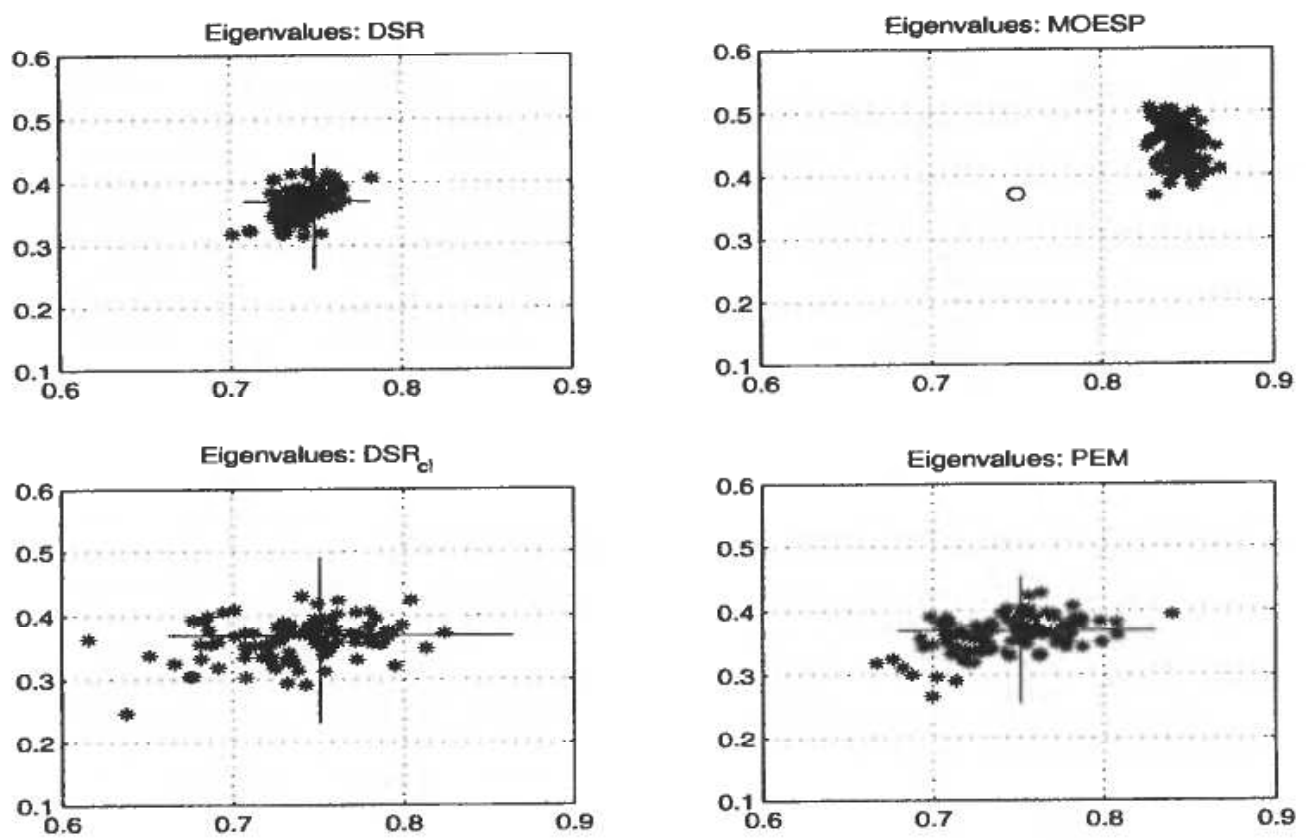

Figure 11. The pole estimates from the closed loop data as described in Example 6.4 with reference signal $r_{k}=\sin (k / 1.526)$. The control system is as in Figure 1.

The states are not needed in order to compute the extended observability matrix and, hence, to identify the system dynamics, i.e., the number of states $n$ and the system matrices $A$ and $D$.

An additional condition for a consistent state estimate is that the past horizon $J$ has to tend to infinity. Furthermore, for colored input signals both the extended observability matrix $O_{L}$ and the lower block triangular Toepliz matrix $H_{L}^{d}$ has in general to be known in order to properly computing the states.

The stochastic part of the model, i.e., the Kalman filter gain matrix and the innovations covariance matrix can be identified directly from the data, i.e. from the projection matrix $Z_{J \mid L+1}^{s}$, without solving any Riccati or Lyapunov matrix equations.

The deterministic part of the model can be identified from an optimal least squares problem defined from the projection matrix $Z_{J \mid L+1}^{d}$, the extended observability matrix $O_{L+1}, A$ and $D$.

Furthermore the necessary projections, $Z_{J \mid L+1}, Z_{J \mid L+1}^{d}$ and $Z_{J \mid L+1}^{s}$, which are needed in order to compute a complete state space model realization for the sixtuple matrices $(A, B, D, E, C, F)$ (and/or $K$ and $\left.\Delta=E\left(\varepsilon_{k} \varepsilon_{k}^{T}\right)\right)$, can be computed through a numerically stable LQ decomposition of

$$
\left[\begin{array}{l}
U_{J \mid L+g} \\
U_{0 \mid J} \\
Y_{0 \mid J} \\
Y_{J \mid L+1}
\end{array}\right]
$$

However, it is in general faster to compute $Z_{J \mid L+1}, Z_{J \mid L+1}^{d}$ and $Z_{J \mid L+1}^{s}$ directly from the definitions. This means that the algorithm both can be implemented as an correlation based method and a square root based method. 


\section{References}

Di Ruscio, D. (1997). A method for identification of combined deterministic stochastic systems. In: Applications of Computer Aided Time Series Modeling, Lecture Notes in Statistics 119, Eds. M. Aoki and A. M. Havenner, Springer Verlag, ISBN 0-387-94751-5.

Di Ruscio, D. (1997b). On Subspace Identification of the Extended Observability Matrix. In the proceedings of the 36th Conference on Decision and Control 1997, San Diego, California, December 6-114

Di Ruscio, D. (1996). Combined Deterministic and Stochastic System Identification and Realization: DSR-a subspace approach based on observations. Modeling, Identification and Control, Vol. 17, No.3.

Di RusCio, D. (1995). A method for identification of combined deterministic stochastic systems: robust implementation. The Third European Control Conference ECC95, September 5-8, Rome, Italy.

LARImOKe, W. E. (1990). Canonical Variate Analysis in Identification, Filtering and Adaptive Control. Proc. of the 29th Conference on Decision and Control, Honolulu, Hawaii, December 1990, pp. 596-604.

KuNG, S. Y. (1978). A new identification and Model Reduction Algorithm via Singular Value Decomposition. Conf. on Circuits, Systems and Computers, Pacific Grove, CA, November 1978 , pp. $705-714$

Van OvfrscheE, P. and De Moor, B. (1994). N4SID: Subspace Algorithms for the Identification of Combined Deterministic Stochastic Systems. Automatica, Vol. 30, No. 1, pp. $75-94$.

VAN OVerscheE, P. and De Moor, B. (1996). Subspace Identification for Linear Systems: theory-implementation-applications. Kluwer Academic Publishers.

VAN Overschee, P. and De Moor, B. (1996b). Closed loop subspace system identification. Internal report. Katholieke Universiteit Leuven. Department Elektrotechniek. ESATSISTA/TR 1996-521.

VAN Overschee, P. and De Moor, B. (1997). Closed loop subspace system identification. In the proceedings of the 36th Conference on Decision and Control 1997, San Diego, California, December 6-14.

Verhagen, M. (1994). Identification of the deterministic part of MIMO state space models given on innovations form from input output data. Automatica, Vol. 30, No. 1, pp. 61-74.

Vinerg, M. (1995). Subspace-Based Methods for the Identification of Linear Time-invariant Systems. Automatica, Vol. 31, No. 12, pp. 1835-1851. 